\title{
Bovine breed-specific augmented reference graphs facilitate accurate sequence read mapping and unbiased variant discovery
}

Danang Crysnanto ${ }^{*}$ (D) and Hubert Pausch

\author{
* Correspondence: danang. \\ crysnanto@usys.ethz.ch \\ Animal Genomics, ETH Zürich, \\ Zürich, Switzerland
}

\begin{abstract}
Background: The current bovine genomic reference sequence was assembled from a Hereford cow. The resulting linear assembly lacks diversity because it does not contain allelic variation, a drawback of linear references that causes reference allele bias. High nucleotide diversity and the separation of individuals by hundreds of breeds make cattle ideally suited to investigate the optimal composition of variationaware references.

Results: We augment the bovine linear reference sequence (ARS-UCD1.2) with variants filtered for allele frequency in dairy (Brown Swiss, Holstein) and dual-purpose (Fleckvieh, Original Braunvieh) cattle breeds to construct either breed-specific or pangenome reference graphs using the $\mathrm{vg}$ toolkit. We find that read mapping is more accurate to variation-aware than linear references if pre-selected variants are used to construct the genome graphs. Graphs that contain random variants do not improve read mapping over the linear reference sequence. Breed-specific augmented and pan-genome graphs enable almost similar mapping accuracy improvements over the linear reference. We construct a whole-genome graph that contains the Herefordbased reference sequence and 14 million alleles that have alternate allele frequency greater than 0.03 in the Brown Swiss cattle breed. Our novel variation-aware reference facilitates accurate read mapping and unbiased sequence variant genotyping for SNPs and Indels.

Conclusions: We develop the first variation-aware reference graph for an agricultural animal (https://doi.org/10.5281/zenodo.3759712). Our novel reference structure improves sequence read mapping and variant genotyping over the linear reference. Our work is a first step towards the transition from linear to variation-aware reference structures in species with high genetic diversity and many sub-populations.
\end{abstract}

Keywords: Variation-aware genome graph, Sequence variant genotyping, Reference allele bias 


\section{Introduction}

A reference sequence is an assembly of digital nucleotides that are representative for a species' genetic constitution. Discovery and genotyping of polymorphic sites from whole-genome sequencing data typically involve reference-guided alignment and genotyping steps that are carried out successively [1]. Variants are discovered at positions where aligned sequencing reads differ from corresponding reference nucleotides. Longread sequencing and sophisticated genome assembly methods enabled spectacular improvements in the quality of linear reference sequences particularly for species with gigabase-sized genomes [2]. Recently generated de novo assemblies exceed in quality and continuity all current reference sequences [3, 4]. However, modifications and amendments to existing linear reference sequences causes shifts in their coordinates that require large efforts from the genomics community to make data compatible with updated reference sequences [5].

Domestication and selection for beef and milk production under various environmental conditions have led to the formation of more than thousand breeds of cattle (Bos taurus) with distinct genetic characteristics and high allelic variation within and between breeds [6]. The 1000 Bull Genomes Project discovered almost 100 million sequence variants that are polymorphic in 2700 cattle from worldwide cattle breeds [7, 8]. Nucleotide diversity is higher in cattle than human populations [7, 9]. Yet, all bovine DNA sequences are aligned to the linear consensus reference sequence of a highly inbred Hereford cow to facilitate reference-guided variant discovery and genotyping [10, 11]. A genome-wide alignment of DNA fragments from a B. taurus individual differs from the Hereford-based reference sequence at between 7 and 8 million singlenucleotide polymorphisms (SNPs) and small $(<50 \mathrm{bp})$ insertions and deletions (Indels) $[12,13]$. The number of differences is higher for DNA samples with greater divergence from the reference $[14,15]$.

The bovine linear reference sequence lacks allelic variation and nucleotides that might segregate at high frequency in animals from breeds other than Hereford. Lack of allelic diversity is an inherent drawback of linear reference sequences because it causes reference allele bias. DNA sequencing reads that contain only alleles that match corresponding reference nucleotides are more likely to align correctly than DNA fragments that also contain non-reference alleles $[16,17]$. Reads originating from DNA fragments that are highly diverged from corresponding reference nucleotides will either obtain low alignment scores, or align at incorrect locations, or remain un-mapped [18]. Reference bias compromises analyses that are sensitive to accurately mapped reads and prevents the precise estimation of allele frequencies [16, 19-21].

Graph-based [17, 22] and personalized reference genomes [5, 23] mitigate reference allele bias. Existing linear reference coordinates can serve as backbones for variationaware genome graphs. Nodes in the graph represent alleles at sites of variation and edges connect adjacent alleles. Once a variation-aware genome graph contains all alleles at known polymorphic sites, every haplotype can be represented as a walk through the graph [24]. However, an optimal balance between graph density and computational complexity is key to efficient whole-genome graph-based variant analysis because adding sites of variation to the graph incurs computational costs. Recently, Pritt et al. [18] developed the FORGe software tool to prioritize variants for graph genomes. Their results provide a framework to build genome graphs that enable read mapping accuracy 
improvements over linear references at tractable computational complexity. A genome graph-based sequence analysis workflow is implemented in the variation graph toolkit ( $v g$, https://github.com/vgteam/vg) [22]. The $v g$ toolkit enables the mapping of sequence reads to variation-aware graphs that incorporate linear reference coordinates as a backbone. It also facilitates to augment genome graphs with genetic variants that have more complex topology (e.g., duplications, inversions, and translocations) [25]. Graph-based references have been investigated primarily in humans and species with small genome sizes [17]. High nucleotide diversity and the separation of individuals by breeds make cattle an ideally suited species to investigate the optimal composition of reference graphs for gigabase-sized genomes.

Here, we investigate sequence read mapping and variant genotyping accuracy using variation-aware reference structures in cattle. Using sequence variant genotypes of 288 cattle from four dairy and dual-purpose breeds, we construct breed-specific augmented and pan-genome reference graphs using the $v g$ toolkit [22]. We prioritize sequence variants to be added to the graphs and assess accuracy of read mapping for variation-aware and linear references (Fig. 1). We show that breed-specific augmented and pan-genome graphs allow for significant read mapping accuracy improvements over linear reference sequences. We also construct a bovine whole-genome reference graph and show that unbiased and accurate sequence variant genotyping is possible from this novel

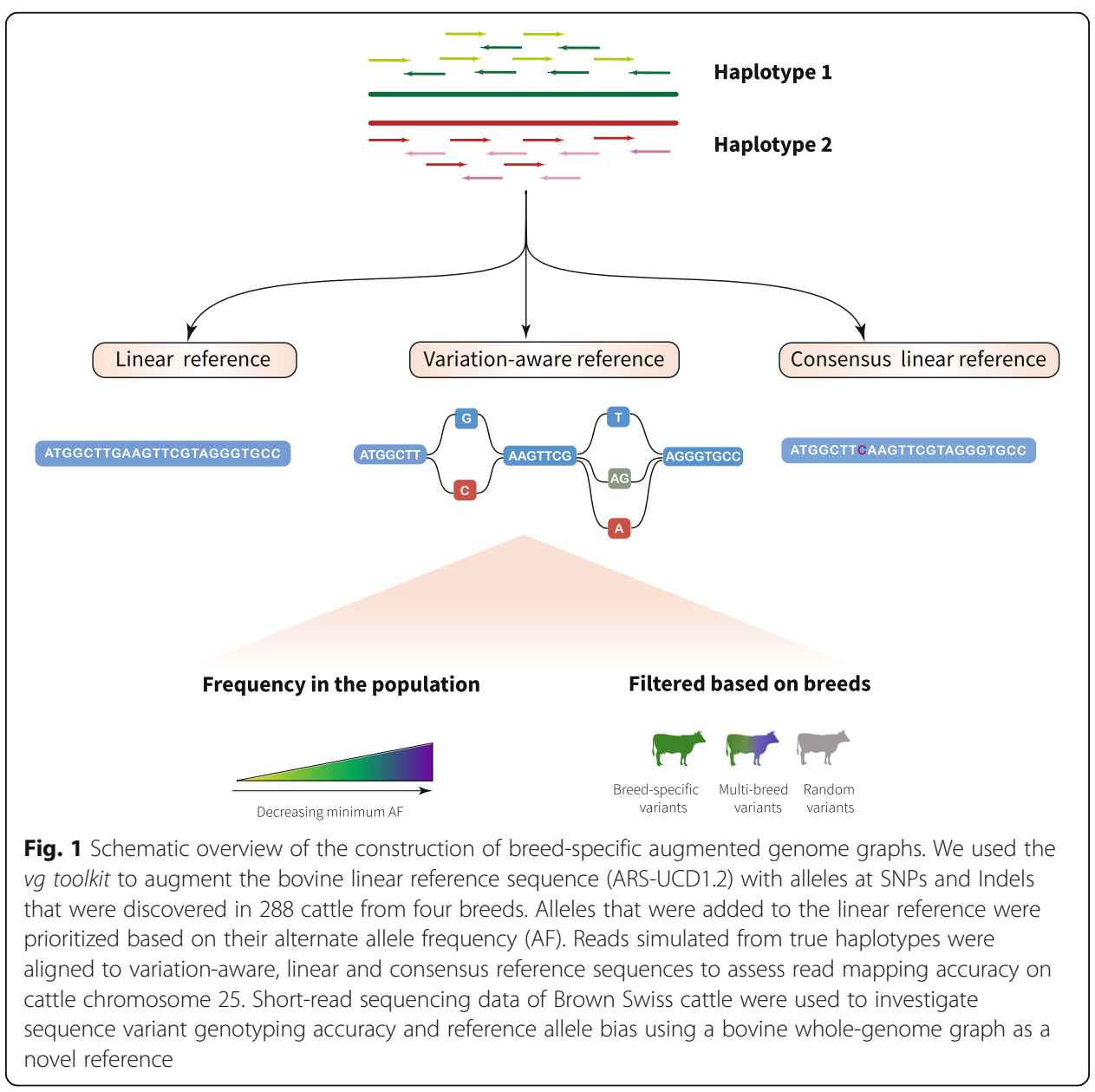


reference structure. Together, we hope that our study can serve as a first step towards the transition from linear to variation-aware references in species with high genetic diversity and many sub-populations.

\section{Results}

\section{Construction of bovine breed-specific augmented genome graphs}

Breed-specific augmented reference graphs were constructed for four genetically distinct dairy (Brown Swiss (BSW), Holstein (HOL)) and dual-purpose (Fleckvieh (FV), Original Braunvieh (OBV)) cattle breeds using the Hereford-based linear reference sequence (ARS-UCD1.2) of chromosome 25 as a backbone (Fig. 2a). Average nucleotide diversity ( $\pi$ ) estimated using 295,801 (HOL), 336,390 (FV), 347,402 (BSW), and 387,855 (OBV) biallelic variants of chromosome 25 ranged from 0.00177 (BSW) to 0.0019 (OBV) for the four breeds (Fig. 2b). To determine the optimal composition of bovine variation-aware references, we augmented the linear reference of chromosome 25 with an increasing number of variants (SNPs and Indels) that were filtered for alternate allele frequency in $82 \mathrm{BSW}, 49 \mathrm{FV}, 49 \mathrm{HOL}$, and $108 \mathrm{OBV}$ cattle. In total, we constructed 20 variation-aware graphs for each breed that contained between 2046 (variants had
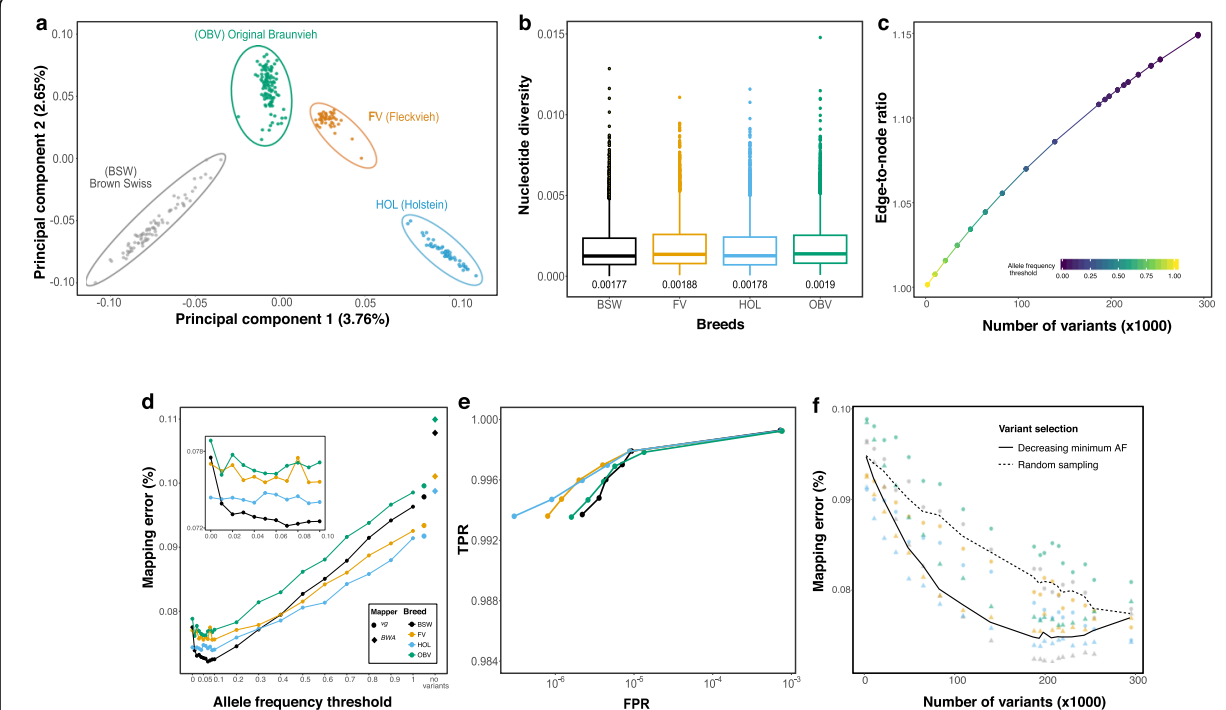

Fig. 2 Accuracy of mapping simulated paired-end reads to genome graphs that contained variants filtered for allele frequency at chromosome 25. a The top principal components of a genomic relationship matrix constructed from whole-genome sequence variants reflect the genetic diversity of the four cattle breeds considered. $\mathbf{b}$ Nucleotide diversity of the four breeds calculated in non-overlapping 10-kb windows for variants of chromosome 25. The values below each boxplot indicate the nucleotide diversity for the four breeds averaged across all sliding-windows. c Edge-to-node ratio of graphs that contained between 2046 and 293,804 variants filtered for allele frequency. $\mathbf{d}$ Proportion of incorrectly mapped reads for four breedspecific augmented genome graphs. Diamonds and large dots represent values from linear mapping using $B W A$ mem and $v g$, respectively. The inset represents a larger resolution of the mapping accuracy for alternate allele frequency thresholds less than 0.1. e True-positive (sensitivity) and false-positive mapping rate (specificity) parameterized on mapping quality of the best performing graph from each breed. $\mathbf{f}$ Read mapping accuracy for breed-specific augmented graphs that contained variants that were either filtered for alternate allele frequency (triangles) or sampled randomly (circles) from all variants detected within a breed. The dashed and solid line represents the average proportion of mapping errors across four breeds using random sampling and variant prioritization, respectively. Colors indicate values obtained for different breeds. Results for single-end mapping are presented in Additional file 1: Fig. S2 
alternate allele frequency $>0.9$ ) and 293,804 (no alternate allele frequency threshold) alleles.

The graph-based representation of bovine chromosome 25 (42,350,435 nucleotides) had 1,323,451 nodes and 1,323,450 edges. The number of nodes increased proportionally with the number of variants added to the reference. When we added a maximum number of 293,804 variants to the linear reference sequence of chromosome 25 , the variation-aware graph contained 2.02 million nodes. The number of edges increased faster than the number of nodes, ranging from 1.32 (empty) to 2.33 (293,804 variants included) million. Consequently, the edge-to-node ratio increased when variants were added to the graph (Fig. 2c). The number of paths through a graph grows rapidly with the number of variants being added to the graph. The index for the chromosome 25 reference graph contained 84.69 and 118.82 million $k$-mers $(k=256)$ when 2046 and 293,804 variants, respectively, were added to the graphs (Additional file 1: Fig. S1).

\section{Variant prioritization based on allele frequency}

We simulated 10 million paired-end reads $(2 \times 150 \mathrm{bp})$ corresponding to approximately 35 -fold coverage of bovine chromosome 25 from haplotypes of BSW, FV, HOL, and OBV cattle. Using either BWA mem or $v g$, we mapped the simulated reads to the respective breed-specific augmented reference graphs and the linear reference sequence. Variants that were only detected in animals used for read simulation were not added to the breed-specific augmented genome graphs. We observed fewer mapping errors using $v g$ than $B W A$ mem when simulated reads were aligned to a linear reference sequence. This finding was consistent for the four breeds investigated (Fig. 2d). Variation-aware references that contained variants filtered for allele frequency in the respective breed reduced the mapping errors for all breeds. The proportion of reads with mapping errors decreased significantly with the number of variants added to the genome graph (Fig. 2d, Pearson $R=0.94, P<10^{-16}$ ).

Read mapping accuracy increased almost linearly between alternate allele frequency threshold 1 and 0.1, i.e., until 186,680 variants with allele frequency greater than 0.1 were added to the graph (Pearson $R=0.94, P<10^{-16}$ ). Adding additional alleles that had alternate allele frequency between 0.1 and 0.01 to the graphs did not further improve read mapping accuracy over the scenario with an alternate allele frequency threshold of 0.1 ( $P=0.13$, Fig. $2 \mathrm{~d}$ inset). Read mapping accuracy declined (particularly in BSW) when the graphs contained rare alleles (alternate allele frequency $<0.01$ ) likely because such alleles are not observed in most animals of a population. Maximum read mapping accuracy was achieved at allele frequency thresholds between 0.2 and 0.01 , when the graphs contained between 139,322 and 293,628 variants filtered for allele frequency. The number of erroneously mapped reads was clearly higher for graphs that contained randomly sampled than prioritized variants (Fig. 2f). This finding corroborates that variant prioritization based on alternate allele frequency is important to achieve high mapping accuracy with graph-based reference structures.

We also applied the methods implemented in the FORGe software [18] to prioritize variants for the breed-specific augmented graphs (Additional file 2: Note S1). It turned out that genome graphs that were constructed with variants selected by the Pop Cov strategy, which relies solely on variant frequency information, enabled the highest 
mapping accuracy improvements over the linear reference. For example, we achieved the highest paired-end read mapping accuracy for the Brown Swiss reference graph (0.0722\% erroneously mapped reads) using the Pop Cov method when 208,288 variants were added to the chromosome 25 reference (i.e., the top $60 \%$ of the ranked variants). The prioritized variants correspond to an alternate allele frequency threshold of 0.06 . Variant prioritization approaches that also take into account factors other than allele frequency, e.g., the proximity of a variant to an already added variant in the graph or the repetitiveness of the resulting genome graph, did not lead to additional accuracy improvements.

Read mapping accuracy was highly correlated (Pearson $R=0.94, P<10^{-16}$ ) for singleand paired-end reads (Additional file 1: Fig. S2). However, the accuracy improvement of variation-aware over linear mapping was higher for single- than paired-end reads, possibly because distance and sequence information from paired reads facilitate linear read alignment.

Read mapping accuracy differed significantly among the four breeds analyzed $(P=$ $10^{-15}$, linear model with allele frequency as covariate) although all breed-specific augmented graphs contained the same number of variants at each allele frequency threshold (Fig. 2d). Linear mapping accuracy also differed among the breeds. We observed the highest error rate for reads aligned to the OBV-specific augmented reference graph. In 500 randomly sampled subsets of 35 sequenced cattle per breed, we discovered more sequence variants on chromosome 25 in OBV $(N=305 \pm 5 \mathrm{~K})$ than either FV $(N=$ $291 \pm 3 \mathrm{~K})$, BSW $(N=276 \pm 6 \mathrm{~K})$ or HOL $(N=259 \pm 2 \mathrm{~K})$, reflecting that nucleotide diversity is higher in OBV than the other three breeds, which agrees with a recent study [26]. Across all alternate allele frequency thresholds considered, read mapping was more accurate for HOL than FV and OBV cattle, possibly because both genetic diversity and effective population size is less in HOL than the other breeds considered [27]. At allele frequency thresholds between 0.02 and 0.3 , read mapping was more accurate for BSW than the other breeds. The proportion of variants with alternate allele frequency $>0.02$ was lower for BSW (84.1\%) than the other breeds (86.3-89.2\%). We detected more rare variants (allele frequency less than 0.05) in BSW and OBV than FV and HOL, likely reflecting differences in sample size (Additional file 1: Fig. S3). An excess of singletons and rare variants in BSW and OBV cattle may have contributed to the decline in mapping accuracy at low alternate allele frequency thresholds (Fig. 2d inset, Additional File 3: Table S2). Our findings indicate that differences in nucleotide diversity and allele frequency distributions across populations may affect read mapping accuracy to both linear and breed-specific augmented reference structures.

\section{Comparison between bovine and human genome graphs}

We used publicly available whole-genome sequence variant data from phase 3 of the 1000 Genomes Project [28] to construct genome graphs for four genetically distinct human populations (Fig. 3a, GBR (British, European), YRI (Yoruba Nigeria, African), STU (Sri Lankan Tamil, South Asia), and JPT (Japanese, East Asia)). The effective population size is more than 20-fold higher for the human than cattle populations (e.g., 3100 for JPT and $\sim 7500$ for YRI [29] vs. $\sim 80$ for OBV and $\sim 160$ for FV [30, 31]). While the average number of sequence variants detected per sample was lower for the human 


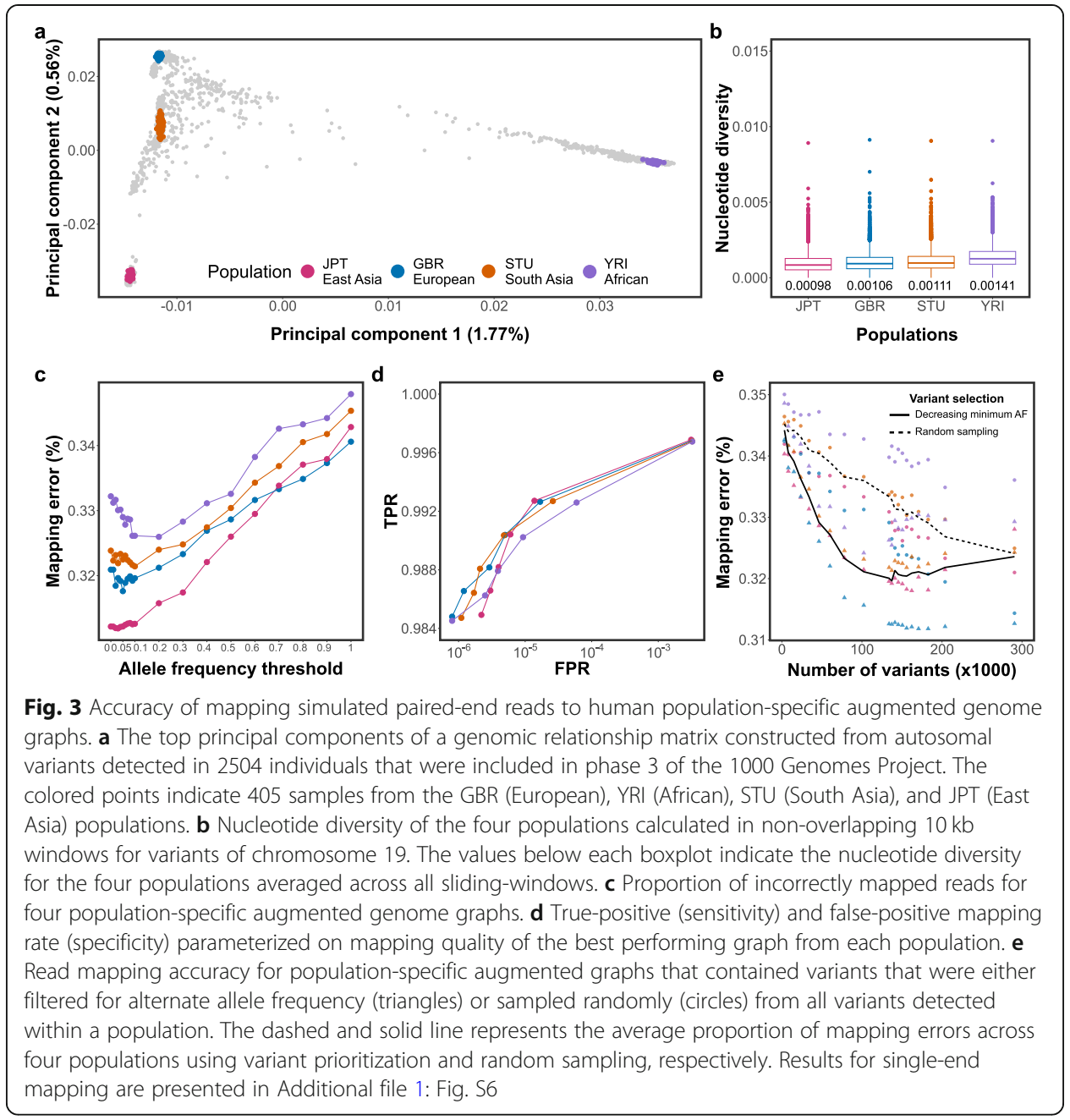

than cattle populations $(4,248,082$ vs. $6,973,036)$, the proportion of singletons is higher in the human than cattle samples $(23.00 \%$ in human vs. $14.01 \%$ in cattle) (Additional file 3: Table S1). The proportion of sequence variants that had minor allele frequency less than 0.05 was between 44.88 and $55.45 \%$ in the four human and between 23.65 and $38.70 \%$ in the four cattle populations (Additional file 1: Fig. S4). Nucleotide diversity ranged from 0.00098 (JPT) to 0.00141 (YRI) (Fig. 3b).

We considered the linear reference sequence of human chromosome 19 (g1k_v37 ref) as a backbone for the human genome graphs because its length $(59,128,983 \mathrm{bp})$ and the number of variants detected per sample was similar to the values for bovine chromosome 25. Genetic diversity and allele frequency distributions were similar using either chromosome 19 or whole-genome variants indicating that the results obtained using chromosome 19 are representative for the human genome (Additional file 1: Fig. S4, S5, Additional file 3: Table S2). To construct population-specific augmented graphs, we used phased genotypes at 291,303, 306,304, 355,107, and 521,021 variants of chromosome 19 that were available for 104 JPT, 91 GBR, 102 STU, and 108 YRI individuals. Once the variants that were only detected in individuals used for simulating reads were removed from the graphs, the population-specific augmented graphs for the GBR, YRI, STU, and JPT populations contained between 3153 (alternate allele 
frequency > 0.9) and 290,593 (no alternate allele frequency threshold) variants. We subsequently simulated 10 million reads from haplotypes of one individual per population and mapped the reads to the respective population-specific augmented genome graphs.

As observed for the bovine breed-specific augmented genome graphs, read mapping accuracy increased almost linearly between alternate allele frequency threshold 1 (no variants included) and 0.1 (133,891 variants added to the graph) (Fig. 3c). Adding lowfrequency variants (alternate allele frequency between 0.01 and 0.1 ) did not further improve the mapping accuracy. Mapping accuracy decreased for all graphs when we added very rare variants and singletons to the graphs. This pattern was most apparent for YRI which had the highest proportion of rare variants and nucleotide diversity among the four populations considered. Read mapping accuracy differed among the four populations analyzed. We observed the lowest number of mis-mapped reads when reads simulated from a JPT individual were aligned to a JPT-specific augmented genome graph. The highest number of mis-mapped reads was observed when reads simulated from a YRI individual were aligned to a YRI-specific augmented genome graph. Mapping accuracy was higher for GBR than STU. These findings indicate that the mapping accuracy is negatively correlated with nucleotide diversity. Mapping accuracy improvements over the linear reference sequence were less when randomly sampled variants were added to the graphs (Fig. 3e).

While the overall pattern of the mapping accuracy improvements over the linear reference was similar for human and bovine genome graphs across all allele frequency thresholds considered, the proportion of mis-mapped paired-end reads was approximately four-fold higher in the human than bovine alignments (two-fold for single-end reads; Additional file 1: Fig. S6). This finding was also apparent when the populationspecific augmented graphs were parameterized on mapping quality to obtain sensitivity and specificity (Fig. 2e and Fig. 3d).

\section{Mapping to breed-specific augmented genome graphs}

Next, we compared read mapping accuracy between bovine breed-specific augmented and pan-genome graphs (i.e., graphs that contained variants filtered for allele frequency across multiple populations) using reads simulated from phased variants of bovine chromosome 25. We constructed four breed-specific augmented genome graphs that contained variants that had alternate allele frequency $>0.03$ in either the BSW, FV, HOL, or OBV breeds. HOL had the lowest number of variants $(N=243,145)$ with alternate allele frequency $>0.03$, reflecting that sample size was lower in HOL than the other breeds. To ensure that the density of information was comparable across all breed-specific augmented graphs, we randomly sampled 243,145 variants with alternate allele frequency $>0.03$ from the BSW, FV, and OBV populations and added them to the respective graphs. The pan-genome graph contained variants that had alternate allele frequency $>0.03$ in 288 individuals from the four populations. The random graph contained 243,145 randomly sampled variants for which haplotype phase and the allele frequency in the BSW, FV, HOL, or OBV breeds was unknown (see the "Methods"

section). To investigate read mapping accuracy, we simulated 10 million sequencing reads ( $150 \mathrm{bp}$ ) from BSW haplotypes and mapped them to the variation-aware and linear reference sequences. Variants that were only detected in the BSW animal used for 
simulating reads were excluded from the graphs. However, in order to determine an upper bound for graph-based read mapping accuracy, we also constructed a "personalized" genome graph, i.e., a graph that contains only haplotypes of the animal used for simulating the reads. We repeated the selection of variants, construction of variationaware graphs and subsequent read mapping ten times.

The average length, number of nodes, number of edges, and edge-to-node ratio of the variation-aware graphs were $42.60 \mathrm{Mb}, 1,907,248,2,155,799$, and 1.13, respectively. Most variants of the random graph $(87.81 \%)$ were not detected at alternate allele frequency $>0.03$ in BSW, FV, OBV, and HOL indicating that they were either very rare or did not segregate in the four breeds considered in our study. Of 243,145 variants, an intersection of $48.13 \%$ had alternate allele frequency greater than 0.03 in the four breeds considered (Fig. 4a). The average number of variants that were specific to the breed-specific augmented graphs ranged from 8010 in BSW to 20,392 in FV.

Personalized genome graphs, i.e., graphs that are tailored to a specific individual, enable the largest read mapping accuracy improvements over linear references. The proportion of mis-mapped reads was $0.0694 \%$ when a personalized BSW graph was used as a reference. Apart from the personalized graph, the highest mapping accuracy, sensitivity, and specificity was achieved when the simulated BSW reads were aligned to a BSW-specific augmented graph (Fig. 4b-d). The proportion of erroneously mapped paired-end reads was $0.073 \%$ for the BSW-specific augmented graph. Sensitivity and specificity were slightly lower and the number of reads with mapping errors was slightly higher when the same reads were aligned to a pan-genome graph. The read mapping accuracy differed only slightly between the breed-specific augmented and pan-genome graph because the overlap of variants that were included in both variation-aware references was high (Additional file 1: Fig. S8). The number of mapping errors was higher (adjusted $P<10^{-16}$, pairwise $t$ test, Additional file 1: Fig. S9) when BSW reads were aligned to genome graphs that contained variants filtered for allele frequency in either the FV, HOL, or OBV populations.

We also simulated reads from haplotypes of FV, HOL, and OBV cattle. Similar to our findings using reads simulated from BSW cattle, mapping was more accurate to breedspecific than either pan-genome graphs or graphs that were augmented with variants filtered for allele frequency in other breeds (Additional file 1: Fig. S10).

Mapping reads to a linear reference sequence using BWA mem with default parameter settings was the least sensitive and least specific mapping approach tested. Linear mapping using $v g$ was also less accurate than variation-aware mapping. This finding indicates that accuracy improvements of variation-aware over linear mapping are attributable to differences in the reference structure rather than mapping algorithms. All graphs that contained pre-selected variants that had alternate allele frequency greater than 0.03 enabled significantly $\left(P=10^{-16}\right.$, two-sided $t$ test) higher mapping accuracy than linear references. This was also true when reads were mapped to graphs that contained variants that were filtered for allele frequency in a different breed, likely because many common variants segregated across the four breeds considered (Fig. 4a).

Recently, Grytten et al. [32] showed that an adjusted linear alignment approach that relies on a combination of $B W A$ mem and Minimap2 [33] may improve linear mapping accuracy because the default setting of $B W A$ mem might miss sub-optimal alignments and overestimate mapping quality for multi-mapping reads [32,34]. We found that this 
a
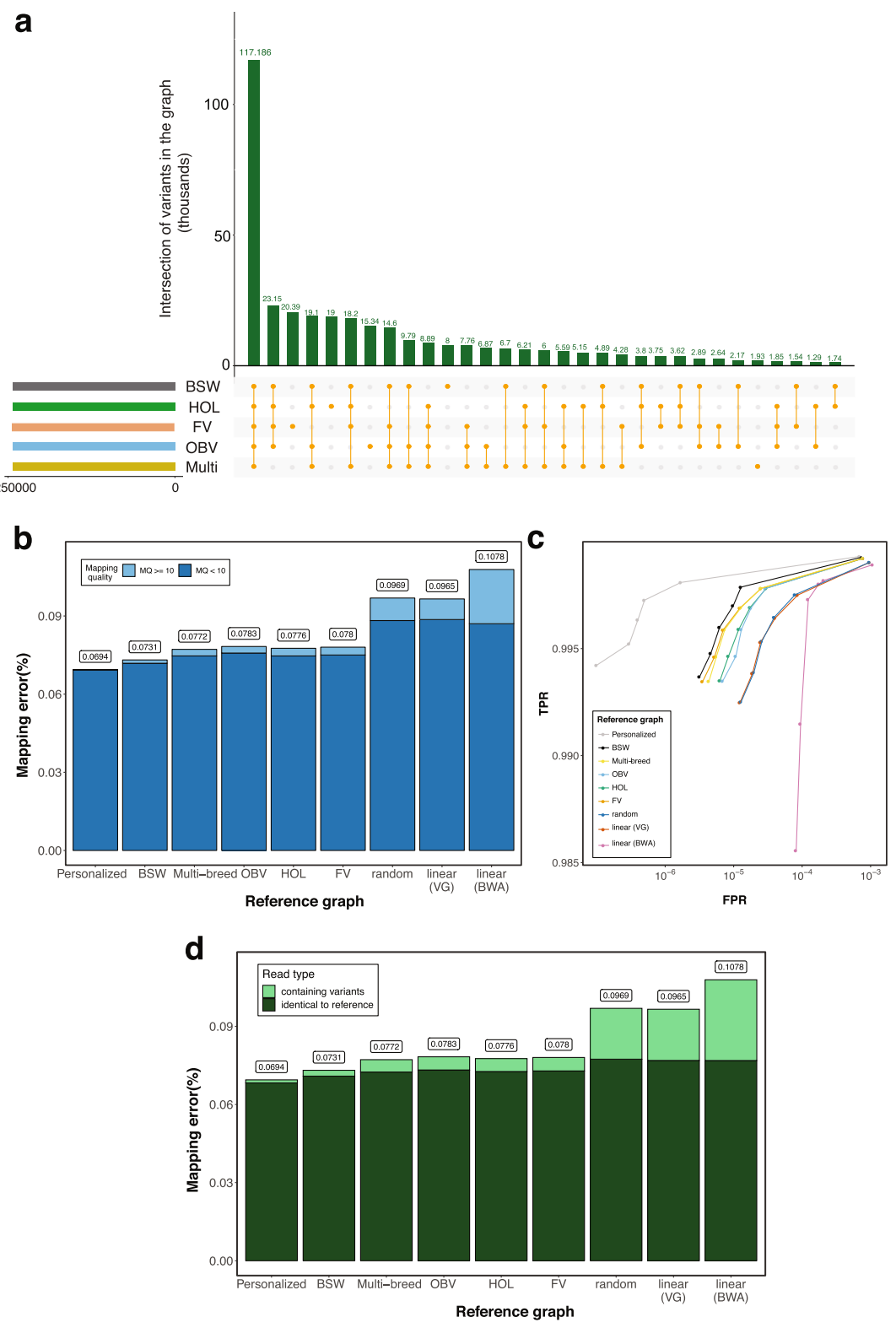

Fig. 4 The accuracy of mapping simulated BSW paired-end reads to variation-aware and linear reference structures. a We added 243,145 chromosome 25 variants to the Hereford-based reference sequence that were filtered for alternate allele frequency $>0.03$ in either the BSW, FV, HOL, or OBV populations. The pangenome graph (Multi) contained 243,145 variants that had alternate allele frequency threshold $>0.03$ across 288 cattle from the four breeds considered. The bars indicate the overlap of variants (averaged across ten replications) that were added to different graphs. b Proportion of simulated BSW reads that mapped erroneously against personalized graphs, breed-specific augmented graphs, pan-genome graphs (Multibreed), random graphs, or linear reference sequences. We used $v g$ and BWA mem for linear mapping. Dark and light blue colors represent the proportion of incorrectly mapped reads that had phred-scaled mapping quality $(\mathrm{MQ})<10$ and $\mathrm{MQ}>10$, respectively. c True-positive (sensitivity) and false-positive mapping rate (specificity) parameterized on mapping quality. $\mathbf{d}$ Proportion of BSW reads that mapped incorrectly against breed-specific augmented graphs, pan-genome graphs (Multi-breed), random graphs, or linear reference sequences. Dark and light green colors represent the proportion of incorrectly mapped reads that matched corresponding reference nucleotides and contained non-reference alleles, respectively. Results for singleend mapping are presented in Additional file 1: Fig. S7 
approach enables to reduce the proportion of mis-mapped from 0.1078 to 0.0983 in cattle (Additional file 2: Note S2). Improved mapping accuracy from the combination of $B W A$ mem and Minimap 2 primarily results from less incorrectly mapped reads that had mapping quality $>10$, indicating a better mapping quality assignment. The mapping accuracy from the adjusted linear alignment approach is similar to the linear mapping accuracy obtained using $v g$ but considerably lower than using breed-specific augmented graphs (Additional file 2: Note S2). The number of paired-end reads with mapping errors is $26 \%$ higher using the adjusted linear alignment approach than breedspecific augmented reference graphs.

Reference graphs that contained random variants, i.e., variants that were neither phased, nor filtered for allele frequency in the breeds of interest, did not improve mapping accuracy, sensitivity and specificity over linear references (adjusted $P=0.74$ and 0.35 for single- and paired-end, pairwise $t$ test, Additional file 1: Fig. S9).

Compared to linear mapping using $B W A$ mem with default parameter settings, the number of mapping errors decreased by 39 and 31\% for single- and paired-end reads, respectively, using a breed-specific augmented reference graph. Extrapolated to wholegenome sequencing data required for a 35 -fold genome coverage, the use of breedspecific augmented reference graphs could reduce the number of incorrectly mapped single- and paired-end reads by 1,300,000 and 220,000, respectively.

Using the BSW-specific augmented graph as a reference, only $1.76 \%$ of the incorrectly mapped reads had mapping quality (MQ) greater than 10 . The MQ of the vast majority (98.24\%) of incorrectly mapped reads was less than 10, i.e., they would not qualify for sequence variant discovery and genotyping using GATK with default parameter settings. The proportion of incorrectly mapped reads with $M Q>10$ was twice as high using either the pan-genome or an across-breed augmented reference graph (3.21$3.85 \%)$. The proportion of incorrectly mapped reads with $\mathrm{MQ}>10$ was higher using either the random graph (8.92\%) or linear reference sequence ( $\mathrm{vg}: 8.19 \%$, BWA mem: $19.3 \%)$.

Of 10 million simulated reads, $19.16 \%$ contained at least one nucleotide that differed from corresponding Hereford-based reference alleles. Using BWA mem, 47.44\% and $28.72 \%$ of the erroneously mapped single- (SE) and paired-end (PE) reads, respectively, contained alleles that differed from corresponding reference nucleotides indicating that incorrectly mapped reads were enriched for reads that contained non-reference alleles (Fig. 4d, Additional file 1: Fig. S7, Additional file 1: Fig. S11). The proportion of erroneously mapped reads that contained non-reference alleles was similar for reads that were aligned to either random (47.62\% and $20.13 \%)$ or empty graphs $(48.20 \%$ and $20.35 \%)$ using $v g$. However, the proportion of incorrectly mapped reads that contained nonreference alleles was clearly lower for the breed-specific augmented (SE: 1.37\%, PE: $3.08 \%$ ) and pan-genome graphs (SE: 2.12\%, PE: 6.14\%). The proportion of incorrectly mapped reads that matched corresponding reference nucleotides was almost identical across all mapping scenarios tested (Fig. 4d, Additional file 1: Fig. S7, Additional file 1: Fig. S11).

Using data from the Ensembl bovine gene annotation (version 99) and RepeatMasker, we determined if the simulated reads originate from either genic regions, interspersed duplications, or low-complexity and simple repetitive regions (Additional file 1: Fig. S12). Regardless of the reference structure used, the mapping accuracy was low for 
reads originating from repetitive regions. Mapping accuracy was higher for reads originating from either genic or exonic regions. Graph-based references enabled more accurate mapping of reads originating from either genic regions or interspersed duplications (including SINEs, LINEs, LTR, and transposable elements) than linear reference sequences. However, graph-based references did not improve the mapping accuracy over linear references for reads that originate from low-complexity or simple repetitive regions.

We further augmented the BSW-specific genome graph with 157 insertion and deletion polymorphisms of bovine chromosome 25 that were detected from short pairedend reads $(2 \times 150 \mathrm{bp})$ of $82 \mathrm{BSW}$ animals using Delly. Adding these variants to the graph either alone or in addition to 243,145 variants that were detected using GATK did not improve the mapping accuracy over the corresponding scenarios that did not include these variants (Additional file 2: Note S3).

\section{Linear mapping accuracy using a consensus reference sequence}

Previous studies reported that linear mapping may be more accurate using populationspecific than universal linear reference sequences $[5,35,36]$. In order to construct bovine linear consensus reference sequences, we replaced the alleles of the chromosome 25 ARS-UCD1.2 reference sequence with corresponding major alleles at 67,142 and 73, 011 variants that were detected in $82 \mathrm{BSW}$ and 288 cattle from four breeds, respectively. Subsequently, we aligned 10 million simulated BSW reads to the linear adjusted sequences using either $v g$ or $B W A$ mem. Read mapping was more accurate to the consensus than original linear reference sequence (Fig. 5, Additional file 1: Fig. S13). The accuracy of mapping was higher when reference nucleotides were replaced by corresponding major alleles that were detected in the target than multi-breed population. However, the mapping of reads was less accurate, sensitive, and specific using either of the consensus linear reference sequences than the breed-specific augmented graphs (Fig. 5b).

\section{Read mapping and variant genotyping using whole genome graphs}

In order to develop a breed-specific augmented reference structure for whole-genome applications, we constructed a BSW-specific augmented whole-genome variation-aware reference graph using 14,163,824 autosomal biallelic variants (12,765,895 SNPs and 1, 397,929 Indels) that had alternate allele frequency greater than 0.03 in 82 BSW cattle. The resulting graph contained 111,511,367 nodes and 126,058,052 edges (an edge-tonode ratio of 1.13) and $6.32 \times 10^{9} 256$-mer paths. We also constructed a linear (empty) whole-genome graph that did not contain allelic variation. Subsequently, we mapped paired-end $(2 \times 150 \mathrm{bp})$ sequencing reads of $10 \mathrm{BSW}$ cattle that had been sequenced at between 6- and 40-fold coverage (Additional file 3 Table S4) to the variation-aware and linear reference sequence using either $v g$ map or BWA mem. The $10 \mathrm{BSW}$ cattle used for sequence read mapping were different to the 82 animals used for variant discovery, graph construction, and haplotype indexing.

$62.19,51.35$ and $49.16 \%$ of the reads aligned perfectly (i.e., reads that aligned with full length (no clipping) and without any mismatches or Indels) to the BSW-specific augmented graph, the empty graph, and the linear reference sequence, respectively (Fig. 6a). 

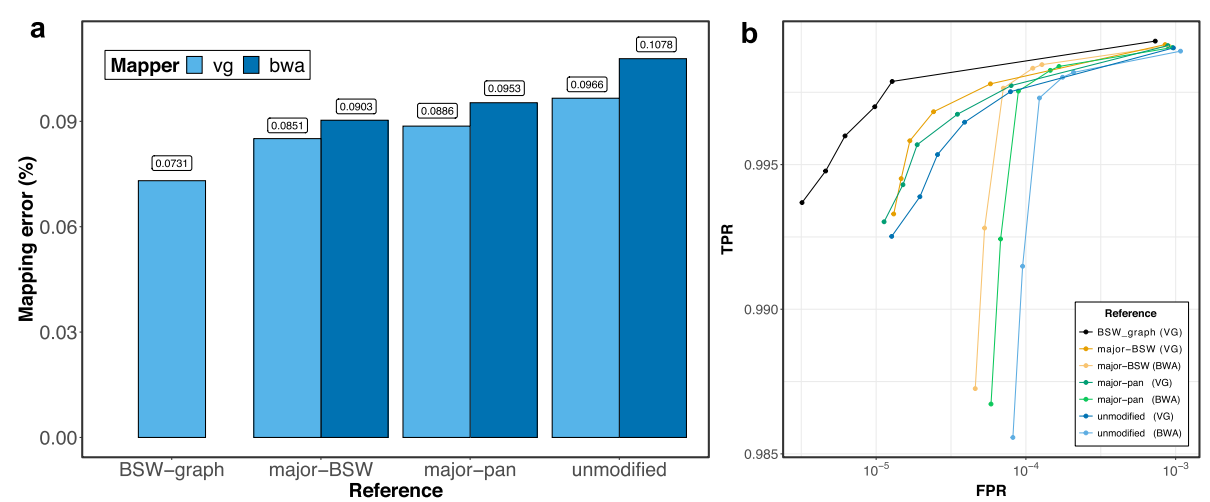

Fig. 5 Paired-end read mapping accuracy using breed-specific augmented genome graphs and consensus linear reference sequences. a Dark and light blue represent the proportion of reads that mapped incorrectly using BWA mem and vg, respectively, to the BSW-specific augmented reference graph (BSW-graph), the BSW-specific (major-BSW) and the multi-breed linear consensus sequence (major-pan) and the bovine linear reference sequence (unmodified). b True-positive (sensitivity) and false-positive mapping rate (specificity) parameterized based on the mapping quality. The results of an analysis where reference nucleotides were only replaced at SNPs is available in Additional file 1: Fig. S13

We observed slightly less uniquely mapped reads using either the whole-genome $(82.46 \%)$ or empty graph $(82.18 \%)$ than the linear reference sequence $(83.18 \%)$ indicating that variation-aware references can increase mapping ambiguity due to providing alternative paths for read alignment.

We converted (surjected) the graph-based read alignments of $10 \mathrm{BSW}$ cattle to corresponding linear reference coordinates and genotyped polymorphic sites using SAMtools mpileup. In order to assess genotyping accuracy, we compared the sequence variant genotypes with array-called genotypes at corresponding positions. Sequence variant genotyping accuracy was correlated with sequencing coverage (Fig. 6b). Genotype concordance, non-reference sensitivity, non-reference discrepancy, and precision did not differ between the graph-based and linear alignments for both raw and hardfiltered genotypes (Fig. 6b, c, Additional file 3: Table S3). The average concordance, precision and recall from the graph-based alignments was 99.76, 99.84, and 98.93,

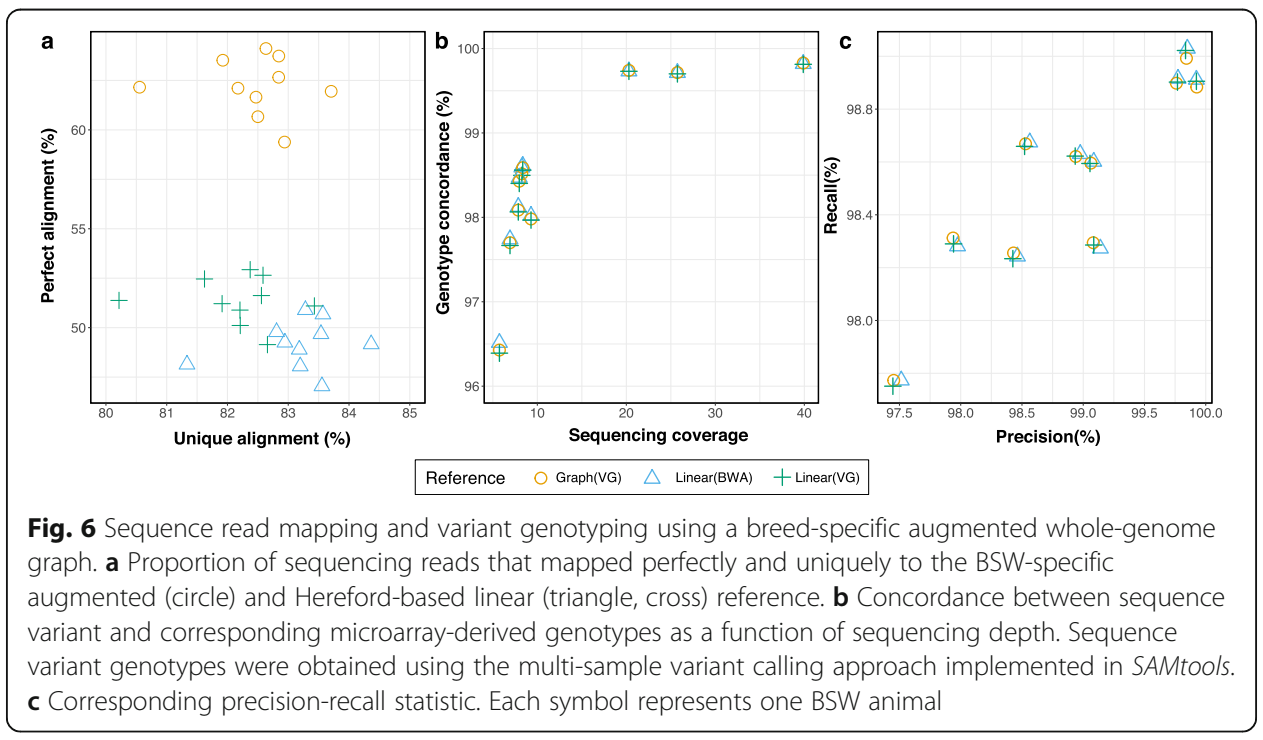


respectively, for three samples (SAMEA6163185, SAMEA6163188, SAMEA6163187) with sequencing coverage greater than 20-fold. We observed similar values for genotypes called using either GATK or Graphtyper (Additional file 3: Table S3). In agreement with our previous findings [12], genotype concordance was slightly higher using Graphtyper, than either SAMtools or GATK.

\section{Variation-aware alignment mitigates reference allele bias}

To investigate reference allele bias in genotypes called from linear and graph-based alignments, we aligned sequencing reads of a BSW animal that was sequenced at 40fold coverage (SAMEA6163185) to either the BSW-specific augmented whole-genome graph or linear reference sequence (Additional file 3: Table S4). We called genotypes using either SAMtools mpileup or GATK. The genotypes were filtered stringently to obtain a high-confidence set of 2,507,955 heterozygous genotypes (2,217,069 SNPs and 290,886 Indels, see the "Methods" section) for reference allele bias evaluation. The BSW-specific augmented whole-genome reference graph contained the alternate alleles at 2,194,422 heterozygous sites (87.49\%).

Using SAMtools to genotype sequence variants from variation-aware and linear alignments, the support for reference and alternate alleles was almost equal at heterozygous SNPs (Fig. 7a), indicating that SNPs are not notably affected by reference allele bias regardless of the reference structure. Alternate allele support decreased with variant length for the linear alignments. As expected, bias towards the reference allele was more pronounced at insertion than deletion polymorphisms. For instance, for 456 insertions that were longer than $30 \mathrm{bp}$, only $26 \%$ of the mapped reads supported the alternate alleles. The allelic ratio of Indel genotypes was closer to 0.5 using graph-based than linear alignments indicating that variation-aware alignment mitigates reference allele bias. However, slight bias towards the reference allele was evident at insertions with length $>12 \mathrm{bp}$, particularly if the alternate alleles were not included in the graph (Fig. 7a). Inspection of the read alignments using the Sequence Tube Map graph visualization tool [37] corroborated that the support for alternate alleles is better using graph-based than linear references (Additional file 1: Fig. S14).

Both the number of reads mapped and the number of mapped reads supporting alternate alleles was higher at Indels using graph-based than linear alignments (Additional file 1: Fig. S15). The difference in the number of mapped reads between graph-based and linear alignments increased with variant length. However, the number of mapped reads supporting the reference alleles did not differ between the graph-based and linear alignments. This finding indicates that reduced reference allele bias at Indel genotypes called from graph-based alignments is due to the improved mapping of reads that contain non-reference alleles.

We next investigated if these conclusions also hold for genotypes called by GATK. While SAMtools mpileup detects variants directly from the aligned reads [38], GATK HaplotypeCaller locally realigns the reads and calls variants from the refined alignments [39]. Using GATK, the allelic ratio was close to 0.5 for genotypes called from graph-based alignments across different lengths of variants that were included in the reference graph (Fig. 7c). However, reference allele bias was evident at insertions that were not included in the reference graph. We also observed an almost equal number of 


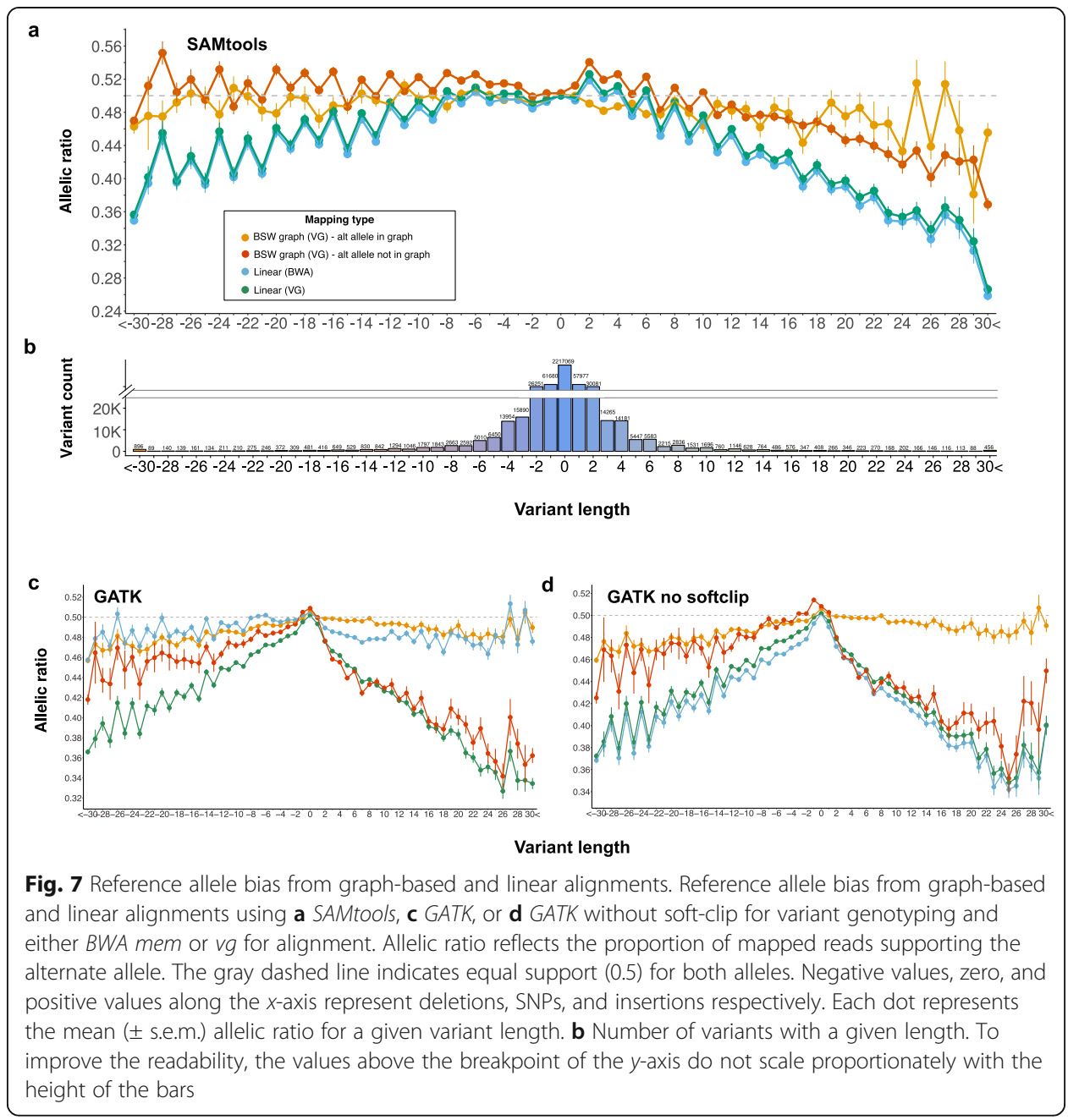

reference and alternate alleles at variants genotyped from linear alignments using GATK. These findings confirm that the local realignment and haplotype-based genotyping approach of GATK might also mitigate reference alleles from linear alignments.

The percentage of soft-clipped reads increased with Indel length in the linear alignments (Additional file 1: Fig. S16). However, the graph-based alignments contained almost no soft-clipped reads across all Indel lengths. In order to investigate the impact of soft-clipping on variant genotyping, we repeated GATK variant discovery and genotyping for the graph-based and linear alignments after all soft-clipped reads were removed (Fig. 7d). As expected, the allelic ratio of genotypes called from the graph-based alignments was not affected by the removal of (very few) soft-clipped reads. However, bias towards the reference allele became evident in genotypes called from linear alignments. This finding confirms that the local realignment of GATK rescues Indels that are initially soft-clipped, thus mitigating reference allele bias. This finding also implies that the original pileup information from graph-based alignments facilitates to confidently detect known Indels while avoiding local realignment as implemented in the GATK HaplotypeCaller. 


\section{Discussion}

To the best of our knowledge, our study is the first to investigate the utility of a variationaware reference for a species with a gigabase-sized genome other than human. We constructed bovine breed-specific consensus sequences and variation-aware reference graphs using a Hereford-based linear reference sequence as backbone and variants that were filtered for allele frequency in four cattle breeds other than Hereford to investigate read mapping accuracy and variant genotyping from different reference structures.

Using sequencing reads simulated from haplotypes of BSW, FV, OBV, and HOL cattle, our findings confirm that a breed-specific consensus sequence improves linear mapping [5, 35]. However, read mapping is less accurate using linear consensus than variation-aware references that contain pre-selected variants. Grytten et al. [32] reported that an adjusted parameter setting of $B W A$ mem and subsequent application of Minimap 2 may further improve the linear mapping accuracy. However, the adjusted linear mapping approach still performs worse than graph-based mapping on reads that contain variants. The accuracy improvements of the adjusted linear mapping approach were small in our study, because the number of sequence variants detected per sample and thus the proportion of reads with variants is almost twice as high in cattle than humans (Additional file 3: Table S1).

Using a bovine variation-aware reference reduced the proportion of erroneously mapped reads by more than $30 \%$ compared to the most widely used linear mapping approach. A similar improvement in mapping accuracy over the linear reference was achieved for a human variation-aware reference genome [18]. The graph-based alignments using the most accurate breed-specific augmented reference graph contained $0.073 \%$ erroneously mapped reads. Incorrectly mapped reads that had high mapping quality $(\mathrm{MQ}>10)$ were less frequent in the graph-based than linear alignments. Thus, a variation-aware reference may reduce the number of flawed genotypes arising from mapping errors that would remain unnoticed due to high mapping quality. Similar to findings in human genome graphs $[18,25]$, bovine variation-aware references did not improve the mapping of short reads that originate from low-complexity regions.

Our findings demonstrate that variant prioritization is key to accurate variationaware read mapping. Based on investigations in four genetically distinct cattle breeds and human populations, we make three important observations: first, variation-aware references that contain random variants for which the allele frequency and haplotype phase in the target populations is unknown do not improve read mapping accuracy over linear references. Our previous study also showed that adding many random variants does barely affect sequence variant genotyping from reference graphs [12]. Adding random unphased variants increases the number of alternative alignment paths that are not necessarily biologically plausible haplotypes, thus increasing mapping ambiguity. Second, read mapping accuracy increases approximately linearly with the number of randomly sampled breed-specific variants being added to the genome graph. Similar findings in the four human population-specific augmented graphs confirm that this observation also holds for populations that are strongly enriched for rare alleles and singletons. Third, the highest mapping accuracy at tractable graph complexity can be achieved when variants filtered for allele frequency are added to the graph. Using variant prioritization approaches that are based on allele frequency, we observed the highest mapping accuracy at allele frequency thresholds between 0.01 and 0.10 in four 
cattle breeds and four human populations. In order to reduce the computational complexity of variation-aware read mapping, previous studies used arbitrarily chosen allele frequency thresholds to prioritize variants to be included in the graphs (e.g., 1\% [22, 40], 5\% [41], 10\% [42]). Using fine-grained allele frequency inclusion thresholds, we find that the read mapping accuracy does not notably differ between the 0.01 and $0.1 \%$ thresholds in most populations. Yet, mapping accuracy declined rapidly for the YRIspecific augmented graph when variants with frequency less than $10 \%$ were added indicating that the optimal inclusion threshold may vary across populations. Variant prioritization approaches that also take into account factors other than allele frequency [18] did not lead to further accuracy improvements in our study. Considering that most cattle breeds have an effective population size between 50 and 200 [43, 44], the vast majority of variants with allele frequency greater than 0.1 can be detected from a few sequenced key ancestor animals [13]. As a matter of fact, key ancestor animals have been sequenced for many cattle breeds [7, 45]. Thus, the construction of variationaware reference structures that are informative for many cattle breeds is readily possible using, e.g., the sequence variant catalog of the 1000 Bull Genomes Project [7, 8].

A pan-genome graph that contained variants filtered for allele frequency across the four cattle breeds enabled almost similar accuracy improvements over the linear reference than breed-specific augmented graphs (Fig. 4b). Although the principal component analysis confirmed that the breeds considered in our study are genetically distinct populations, they share many common alleles. Moreover, compared to human populations, the proportion of rare alleles and singletons is low in cattle. The bovine pangenome graph constructed in our study contained between 75.28 and $80.82 \%$ of the variants that were also added to the breed-specific augmented graphs. Instead of building many breed-specific graphs, the construction of a universal pan-genome graph is likely possible without notably compromising the accuracy of read mapping. This conclusion may hold for many species with genetically distinct sub-populations that share common alleles. Compared to the linear reference, the mapping accuracy was also significantly higher when reads from one breed were mapped to a genome graph that contained variants filtered for allele frequency in another somewhat related breed. Thus, the BSW-specific augmented whole-genome graph constructed in our study will likely improve read mapping accuracy over the linear reference and mitigate reference allele bias also for breeds other than BSW, FV, HOL, and OBV. Our BSW-specific augmented whole-genome graph is available at https://doi.org/10.5281/zenodo.3759712 [46]. In order to facilitate the construction of variation-aware reference structures, the entire workflow to establish whole-genome graphs is also available at https://github. com/danangcrysnanto/bovine-graphs-mapping.

The number of sequencing reads that aligned to the BSW-specific whole-genome graph with full identity increased considerably $(+13 \%)$ over the linear reference sequence at the cost of a slightly reduced $(-0.72 \%)$ number of unique alignments. A twostep graph alignment approach that exploits a refined search space might reduce the number of multiple mappings in dense variation-aware graphs [32]. Compared to a human whole-genome graph, the improvement in perfect mapping over the linear reference was slightly larger in our bovine whole genome graph (9.2\%) [22]. However, the proportion of reads with perfect alignments (62.19\%) was lower in our BSW-specific whole-genome graph, likely because it contained only sequences that were assembled 
to the 29 autosomes. The graph did not contain $269.77 \mathrm{Mb}$ of the sex chromosomes, mitochondrial DNA, and 2180 unplaced contigs. A more sophisticated assembly of the bovine genome with increased continuity particularly at the sex chromosomes [4, 47] might serve as a backbone for an improved variation-aware genome graph.

In order to detect SNPs and Indels from the variation-aware reference graph using widely used sequence variant genotyping methods, we had to make the graph-based alignments compatible with linear coordinates. Thus, our assessment of sequence variant genotyping from the bovine whole-genome graph is based on surjected graph-based alignments. It is possible that converting graph-based to linear alignments compromises variant discovery. However, the accuracy and sensitivity of genotyping did not differ between graph-based and linear alignments indicating that our whole-genome graph facilitates accurate sequence variant (SNPs and small Indels) genotyping. It is worth noting that our analysis considered only SNPs that are located in well-accessible regions of the genome, thus possibly overestimating genotyping accuracy [48, 49]. A benchmark dataset that enables unbiased evaluation of sequence variant genotyping [50] is not available for the four cattle breeds considered in our study. Because approximately $90 \%$ of the considered SNPs were already included in the BSW-specific whole-genome graph, they can be detected and genotyped easily from graph-based alignments [17]. These variants can also be detected and genotyped accurately from linear alignments [12, 51].

As expected, bias towards the reference allele was less in graph-based than linear alignments particularly at variants that were included in the graph. Unbiased genotyping of heterozygous variants from graph-based alignments is possible because reads supporting alternate alleles align better to variation-aware than linear references. Thus, our bovine whole-genome graph offers an appealing novel reference for investigations that either rely on low-coverage sequencing or are sensitive to unbiased allele frequencies $[16,19,52]$. Because a benchmark dataset for an unbiased evaluation of sequence variant genotyping performance [50] is not available in cattle, our assessment was restricted to heterozygous variants that were identified from both linear and graph-based alignments. This set of variants is possibly enriched for variants that can be called confidently from linear alignments, thus underestimating the graph-based genotyping performance (e.g., [22]).

Our study has three limitations. First, variants used to construct the breed-specific augmented genome graphs might be biased because they were detected from linear alignments of short sequencing reads. Variant discovery from an independent variation-aware reference structure might allow for a more complete assessment of genetic variation [50]. Second, we used the Hereford-based linear reference sequence as backbone to construct breed-specific augmented reference sequences. However, the Hereford-based reference sequence might lack millions of basepairs that segregate in the four breeds considered in our study [53-56]. These nucleotides are likely missing in the breed-specific augmented reference graphs constructed in our study. Accurate and continuous genome assemblies from BSW, FV, HOL, and OBV cattle are not available. All bovine genome assemblies that are available to date had been compiled from individuals that are distantly related to the breeds in our study [2, 4, 57]. Haplotyperesolved genome assemblies of cattle from different breeds will facilitate the construction of more informative genome graphs and make non-reference sequences and their sites of variation amenable to genetic investigations $[2,4]$. Third, we did not investigate 
the impact of large sequence variation on sequence read mapping and variant genotyping performance because neither a high-quality benchmark set of large structural variants (cf. [58]) nor long-read sequencing data is available for the four cattle breeds considered. Adding insertion and deletion polymorphisms detected from short-read sequencing data did not lead to accuracy improvements in our study likely because structural variants detected from short reads are notoriously biased and incomplete [59]. Recent studies indicated that large structural variants can be identified accurately from genome graphs [25, 60-62]. Eventually, a bovine genome graph that unifies multiple breed-specific haplotype-resolved genome assemblies and their sites of variation might provide access to sources of variation that are currently neglected when short sequencing reads are aligned to a linear reference sequence [63-65].

\section{Conclusions}

We constructed the first variation-aware reference graph for Bos taurus that improves read mapping accuracy over the linear reference sequence. The use of this novel reference structure facilitates accurate and unbiased sequence variant genotyping. Our results indicate that the construction of a widely applicable bovine pan-genome graph is possible that enables accurate genome analyses for many diverged breeds.

\section{Methods}

\section{Whole-genome sequencing data}

We used short paired-end sequencing reads of 288 cattle from dairy $(n=82$ Brown Swiss (BSW), $n=49$ Holstein (HOL)) and dual-purpose ( $n=49$ Fleckvieh (FV), $n=108$ Original Braunvieh $(\mathrm{OBV})$ ) breeds to detect variants that segregate in these populations. The average sequencing depth of the 288 cattle was 12.71 -fold, and it ranged from 3.49 to 70.04. Most of the sequencing data were generated previously [7, 12, 13, 66, 67]. Accession numbers for all animals are available in Additional file 3: Table S4.

We trimmed adapter sequences from the raw data and discarded reads for which the phred-scaled quality was below 15 for more than $15 \%$ of the bases using fastp (Chen at al., 2018). Subsequently, the sequencing reads were aligned to the linear reference assembly of the bovine genome (ARS-UCD1.2, GCF_002263795.1) using BWA mem [34]. Duplicates were marked and the aligned reads were coordinate sorted using the Picard tools software suite (http://broadinstitute.github.io/picard) and Sambamba [68], respectively. We discovered and genotyped polymorphic sites from the linear read alignments using the Best Practices Workflow descriptions for multi-sample variant calling with GATK (version 4.1.0) [1]. Because a truth set of variants required for variant quality score recalibration (VQSR) is not available for Bos taurus, we followed the recommendations for sequence variant discovery and filtration when applying VQSR is not possible. Genotypes of the hard-filtered variants were subsequently refined, and sporadically missing genotypes were imputed with $B E A G L E$ v4 [69] using the genotype likelihoods from the GATK HaplotypeCaller model as input values. Additional information on the sequence variant genotyping workflow and the expected genotyping accuracy can be found in Crysnanto et al. [12]. Nucleotide diversity was calculated in nonoverlapping $10 \mathrm{~kb}$ windows separately for each breed using the $\pi$ (nucleotide diversity) module implemented in the vcftools software [70]. 
We discovered and genotyped large structural variants ( $>50 \mathrm{bp}$ ) including insertions, deletions, inversions, duplications, and translocations in 82 sequenced BSW animals using Delly v0.7.8 [71] with the default settings. We retained only insertion and deletion variants that had been refined using split-reads (PRECISE-flag in the vcf file).

The principal components of a genomic relationship matrix constructed from wholegenome sequence variant genotypes were calculated using PLINK v1.9 [72]. The top principal components separated the animals by breeds, corroborating that the four breeds are genetically distinct (Fig. 2a). To take haplotype diversity and different linkage disequilibrium phases across breeds into account, the sequence variant genotypes were phased for each breed separately using BEAGLE v5 [73].

Unless stated otherwise, our analyses included 541,876 biallelic SNPs and Indels that were detected on bovine chromosome 25. The vg toolkit version 1.17.0 "Candida" [22] was used for all graph-based analyses.

\section{Haplotype-aware simulation of short sequencing reads}

We simulated 10 million reads $(150 \mathrm{bp})$ from reference haplotypes of one animal per breed that had sequencing coverage greater than 20-fold (see Additional file 3 Table S4). Therefore, we added the phased sequence variants of each of the four animals to the linear reference to construct individualized reference graphs using $v g$ construct. The haplotype-aware indexes of the resulting graphs were built using $v g$ index $x g$ and $g b w t$. $v g$ paths and $v g$ mod were used to extract the haplotype paths from the individualized reference graphs. Subsequently, we simulated 2.5 million paired-end reads $(2 \times 150 \mathrm{nt})$ from each haplotype using $v g$ sim, yielding 10 million $150 \mathrm{bp}$ reads per breed corresponding to approximately 35-fold sequencing coverage of bovine chromosome 25 . The simulation parameter setting for read and fragment length was 150 and $500( \pm 50)$, respectively. The substitution and indel error rate was 0.01 and 0.002 , respectively, according to the settings used in Garrison et al. [22].

\section{Read mapping to graphs augmented with variants filtered for allele frequency}

The alternate allele frequency of 541,876 variants of bovine chromosome 25 was calculated separately for the BSW, FV, HOL, and OBV breeds using sequence variant genotypes of $82,49,49$, and 108 sequenced cattle, respectively. We added to each breedspecific genome graph 20 sets of variants that were filtered for alternate allele frequency using thresholds between 0 and 1 with increments of 0.01 and 0.1 for frequency below and above 0.1, respectively. For instance, at an alternate allele frequency threshold of 0.05 , the graph was constructed with variants that had alternate allele frequency greater than $5 \%$. Alleles that were only detected in the four animals used to simulate reads (see above) were not added to the breed-specific augmented genome graphs.

The four breed-specific augmented genome graphs contained the same number of variants at a given allele frequency threshold to ensure that their density of information was similar. The number of variants added to the graphs was determined according to the breed in which the fewest variants were detected at a given allele frequency threshold. For the other three breeds, we sampled randomly from all variants that were detected at the respective alternate allele frequency threshold. We indexed the breedspecific augmented graphs using $v g$ index to obtain the topological $(x g)$, query $(g c s a)$, 
and haplotype $(g b w t)$ index. Eventually, the simulated reads were aligned to the breedspecific augmented reference graphs using $v g$ map with default mapping parameter settings considering both graph $(x g, g c s a)$ and haplotype $(g b w t)$ indexes.

To compare the accuracy of read mapping between variation-aware and linear reference structures, the simulated reads were also aligned to the linear reference sequence of bovine chromosome 25 using either BWA mem with default parameter settings or $v g$ map. To enable linear mapping with vg map, we constructed an empty graph (without adding any sequence variants) from the linear reference sequence.

\section{Read mapping to human population-specific augmented genome graphs}

We downloaded phased whole-genome variants of 2504 individuals from phase 3 of the 1000 Genomes Project [28] as well as the corresponding reference sequence (g1k_v37; https://www.internationalgenome.org/category/reference/). We selected four populations which we considered to be genetically distinct based on the results of a principal components analysis and for which the number of individuals was similar to the number of individuals for the four cattle breeds, i.e., GBR (British in England and Scotland, European), YRI (Yoruba in Ibadan Nigeria, African), JPT (Japanese in Tokyo, East Asia), and STU (Sri Lankan Tamil, South Asia). The principal components were calculated from a genomic relationship matrix constructed using 81.27 million autosomal variants using the PLINK (v1.9) software [72]. Alternate allele frequency was calculated separately for the four populations for all variants of human chromosome 19. Nucleotide diversity was calculated with the vcftools software as detailed above. In order to construct population-specific augmented genome graphs, we used the reference sequence (g1k v37) of human chromosome 19 as a backbone and added variants filtered for alternate allele frequency in the four populations (following the approach explained above). For each population, we constructed 20 graphs that contained between 3153 and 290,593 variants. We simulated 10 million paired-end reads for each population from reference haplotypes (as detailed above) of four selected samples (GBR: HG00096, YRI: NA18486, JPT: NA18939, STU: HG03642). The simulated reads were then mapped to the population-specific augmented genome graphs using the $v g$ toolkit.

\section{Read mapping to bovine breed-specific augmented graphs}

We simulated 10 million reads from the haplotypes of a BSW animal (SAME A6272105) and mapped them to variation-aware reference graphs that were constructed using variants (SNPs and Indels) filtered for alternate allele frequency greater than 0.03. Alleles that were only detected in SAMEA6272105 were excluded from the graphs. All graphs contained 243,145 variants. The number of variants was determined according to the HOL cattle breed because the lowest number of variants segregated at an alternate allele frequency greater than 0.03 in that breed. To investigate the utility of targeted genome graphs, we mapped the simulated BSW reads to a graph that contained variants filtered for allele frequency in BSW cattle. To investigate across-breed mapping, we mapped the simulated BSW reads to graphs that contained variants filtered for allele frequency in either FV, HOL, or OBV cattle. We also mapped the BSW reads to a bovine pan-genome graph that contained variants that were filtered for allele frequencies across the four cattle breeds. Additionally, we investigated the accuracy of 
mapping reads to a graph that was built from randomly selected variants. To construct the random graph, we randomly sampled from 2,294,416 variants that were detected on bovine chromosome 25 from animals of various breeds of cattle (http://www.1 000bullgenomes.com/doco/ARS1.2PlusY_BQSR_v2.vcf.gz). The allele frequencies and haplotype phases of the random variants were not known. We constructed personalized graphs that contained only variants and haplotypes that were detected in the animals used for read simulation. The variation-aware graphs were subsequently indexed using $v g$ index (see above). The simulated BSW reads were mapped to the different graphs using $v g$ map (see above). The construction and indexing of graphs as well as read simulation and mapping were repeated ten times. We report in the main part of the paper the average values of ten replicates. This entire procedure was repeated with reads that were simulated from the haplotypes of FV (SAMN02671626), HOL (SAMN02671584), and OBV animals (SAMEA5059743).

\section{Read mapping to consensus reference sequences}

We modified alleles of the ARS-UCD1.2 linear reference sequence using the vcf2diploid tool [52]. We created two adjusted linear reference sequences for bovine chromosome 25 :

- major-BSW: 67,142 nucleotides of the linear reference sequence were replaced with the corresponding major alleles detected in $82 \mathrm{BSW}$ cattle.

- major-pan: 73,011 nucleotides of the linear reference sequence were replaced with the corresponding major alleles detected in 288 cattle from four breeds.

Ten million BSW reads were simulated (see above) and mapped to the original and modified linear reference sequences, as well as the corresponding variation aware reference structures using either $B W A$ mem or $v g$ map (see above) with default parameter settings. Since the replacement of reference alleles with Indels causes a shift in the reference coordinate system, we converted the coordinates of simulated reads between the original and modified reference using a local instance of the UCSC liftOver tool [74] that was guided using a chain file produced by vcf2diploid. In order to prevent possible errors arising from coordinate shifts when reference nucleotides are either deleted or inserted at Indels, we repeated the analysis when only the alleles at SNPs were replaced.

\section{Assessment of the read mapping accuracy}

We used $v g$ stats to obtain the number of nodes and edges, biologically plausible paths and length for each variation-aware reference graph. To assess the accuracy of graphbased alignment, we converted the Graph Alignment Map (GAM)-files to JavaScript Object Notation (JSON)-files using vg view. Subsequently, we applied the commandline JSON processor $j q$ (https://stedolan.github.io/jq/) to extract mapping information for each read. Mapping information from linear alignments were extracted from the Binary Alignment Map (BAM)-files using the Python module pysam (version 0.15.3) (https://github.com/pysam-developers/pysam).

Using vg annotate, we annotated the simulated reads with respect to the linear reference coordinates and determined if they contained non-reference alleles. Comparing the true and mapped positions of the simulated reads enabled us to differentiate 
between correctly and incorrectly mapped reads. Following the approach of Garrison et al. [22] and taking into account the possibility that aligned reads may be clipped at Indels, we considered reads as incorrectly mapped if their starting positions were more than $k=150$ ( $k=$ read length) bases distant from true positions. The functional relevance genomic regions where the simulated reads originated from were determined based on the Ensembl annotation (version 99, [75]) of the bovine ARS-UCD 1.2 reference sequence. The coordinates of repetitive elements were determined based on RepeatMasker [76] annotation tables of the UCSC Genome Browser.

In order to assess mapping sensitivity and specificity, we calculated the cumulative TPR (true-positive rate) and FPR (false-positive rate) at different mapping quality thresholds and visualized it as pseudo-ROC (receiver operating characteristic) curve [22] using:

$$
\mathrm{TPR}_{i}=\frac{\sum_{i}^{60} \mathrm{TP}_{k}}{n}
$$

and

$$
\mathrm{FPR}_{i}=\frac{\sum_{i}^{60} \mathrm{FP}_{k}}{n}
$$

where $\mathrm{TP}_{i}$ and $\mathrm{FP}_{i}$ represent the number of correctly and incorrectly mapped reads, respectively, at a given phred-scaled mapping quality threshold $i(60,50,40,30,20,10$, 0 ), and $n$ is the total number of reads mapped.

\section{Read mapping and sequence variant genotyping from bovine whole-genome graph}

Using 14,163,824 autosomal biallelic variants (12,765,895 SNPs and 1,397,929 Indels) that had alternate allele frequency greater than 0.03 in $82 \mathrm{BSW}$ cattle, we constructed a BSWspecific augmented whole-genome graph. The Hereford-based linear reference sequence (ARS-UCD1.2) was the backbone of the graph. Specifically, we constructed graphs for each of the 29 autosomes separately using $v g$ construct. Subsequently, $v g$ ids was run to ensure that the node identifiers were unique in the concatenated whole-genome graph. We removed complex regions from the whole-genome graph using $v g$ prune with default parameter settings and built the topological $(x g)$ and query $(g c s a)$ index for the full and pruned graph, respectively, using $v g$ index. The haplotype paths of the 82 BSW cattle obtained using BEAGLE v5 (see above) were provided using a $g b w t$ index.

To evaluate sequence variant genotyping from the whole-genome graph, we used between $122,753,846$ and $904,047,450$ million paired-end $(2 \times 150 \mathrm{bp})$ sequencing reads from 10 BSW cattle (SAMEA6163185, SAMEA6163188, SAMEA6163187, SAME A6163177, SAMEA6163178, SAMEA6163176, SAMEA6163179, SAMEA6163183, SAMEA6163181, SAMEA6163182, Additional file 3 Table S4) that had been sequenced at between 5.74 and 39.88-fold genome coverage. These animals were not part of the $82 \mathrm{BSW}$ animals that were used to detect the variants that were added to the graph. We trimmed adapter sequences and removed reads that had more than $20 \%$ bases with phred-scaled quality less than 20 using fastp [77]. Subsequently, we mapped the pruned 
reads to either the BSW-specific augmented whole-genome graph or the linear reference sequence using either $v g$ map while supplying both graph $(x g, g c s a)$ and haplotype $(g b w t)$ index to produce GAM files for each sample or BWA mem. To make the coordinates of the graph-based alignments compatible with linear reference coordinates, we converted the GAM- to BAM-files using vg surject. Variants were detected and genotyped from the surjected files using the multi-sample variant calling approach of either GATK [39], Graphtyper [40], or SAMtools [38], as stated above and detailed in [12].

In order to assess the read mapping accuracy from real sequencing data, we calculated the proportion of reads that aligned (i) perfectly and (ii) uniquely [18, 35, 78]. A read was considered to map perfectly if the edit distance was zero along the entire read (NM:0 tag in BWA mem-aligned BAM files; identity 1 in $v g$ map-aligned GAM-files), and without hard clipping ( $\mathrm{H}$ tag) or soft clipping ( $\mathrm{S}$ tag) in CIGAR string. A read was considered to map uniquely if either a single primary alignment was reported for the respective read or reads that had secondary alignments (XA tag in BWA mem-aligned BAM files; secondary_score $>0$ in $v g$ map-aligned GAM-files) had one alignment with phred-scaled mapping quality score of 60 .

The sequenced BSW animals also had Illumina SNP BeadChip-derived genotypes at between 24,512 and 683,752 positions. The sequence variant genotypes were compared to microarray-called genotypes at corresponding positions to calculate recall/non-reference sensitivity, genotype concordance, precision, and non-reference discrepancy [1, 79]. The concordance metrics are explained in Additional file 1: Fig. S17.

Snakemake workflows [80] for whole-genome graph construction, read mapping, and variant discovery are available in the Github repository (https:/github.com/danangcrysnanto/bovine-graphs-mapping).

\section{Assessment of reference allele bias}

Reference allele bias was assessed at the heterozygous genotypes that had been detected in a BSW animal (SAMEA6163185) that had been sequenced at high (40-fold) coverage. Raw sequencing data were filtered as stated above and aligned to either the linear reference sequence or BSW-specific augmented genome graph using BWA mem and $v g$ map, respectively. Sequence variant genotypes were discovered and genotyped from either surjected graph-based or linear alignments using the single sample variant calling approaches implemented in either GATK HaplotypeCaller or SAMtools mpileup. Variants were filtered using quality by depth $(\mathrm{QD})>10$, mapping quality $(\mathrm{MQ})>40$, and minimum read depth (DP) greater than 25 to ensure confident genotype calls and sufficient support for reference and alternate alleles at heterozygous genotypes. We considered only variants that were detected from both graph-based and linear alignments. At each heterozygous genotype, we quantified the number of reads supporting alternate and reference alleles using allelic depth information from the vcf files.

\section{Supplementary information}

Supplementary information accompanies this paper at https://doi.org/10.1186/s13059-020-02105-0.

Additional file 1. Integrated supplementary figures: S1-S17.

Additional file 2. Integrated supplementary notes: S1-S3.

Additional file 3. Integrated supplementary tables: S1-S4.

Additional file 4. Review history 


\section{Acknowledgements}

We thank the many people who made data and software publicly available, particularly vgteam for providing the $\mathrm{vg}$ toolkit as an open source software. We thank Braunvieh Schweiz for providing pedigree and genotype data of Original Braunvieh and Brown Swiss cattle. Semen samples of the sequenced bulls were kindly provided by Swissgenetics.

\section{Review history}

The review history is available as Additional file 4.

\section{Peer review information}

Andrew Cosgrove was the primary editor on this article and managed its editorial process and peer review in collaboration with the rest of the editorial team.

\section{Authors' contributions}

Analyzed data: DC HP; Wrote the paper: DC HP; Read and approved the final version of the paper: DC HP.

\section{Authors' information}

Twitter handles: @crdanang (Danang Crysnanto).

\section{Funding}

This study was supported by a grant from the Swiss National Science Foundation (310030_185229). The sequencing of Original Braunvieh cattle was partially funded from the Federal Office for Agriculture (FOAG), Bern, Switzerland. The sequencing data of the Brown Swiss bulls used for sequence variant genotyping were funded from Swissgenetics. The funders had no role in the design of the study and collection, analysis, and interpretation of data and in writing the manuscript.

\section{Availability of data and materials}

The scripts and data used in this study are available via GitHub repository (https:/github.com/danangcrysnanto/ bovine-graphs-mapping) and archived in Zenodo (data: https://doi.org/10.5281/zenodo.3759712 [46] and scripts: https://doi.org/10.5281/zenodo.3763286 [81]). Raw sequencing read data of 298 cattle used for graph construction, evaluation of variant genotyping accuracy, and assessment of reference allele bias are available at the European Nucleotide Archive (ENA) (http://www.ebi.ac.uk/ena) with study accession of PRJNA238491 [7], PRJEB28191 [12], and PRJEB18113 [67]. Detailed accession numbers for each sample are provided in Additional file 3 Table S4.

\section{Ethics approval and consent to participate}

Not applicable.

\section{Consent for publication}

Not applicable.

\section{Competing interests}

The authors declare that they have no competing interests.

Received: 22 January 2020 Accepted: 14 July 2020

Published online: 27 July 2020

\section{References}

1. DePristo MA, Banks E, Poplin R, Garimella KV, Maguire JR, Hartl C, et al. A framework for variation discovery and genotyping using next-generation DNA sequencing data. Nat Genet. 2011;43:491-8. https://doi.org/10.1038/ng.806.

2. Koren S, Rhie A, Walenz BP, Dilthey AT, Bickhart DM, Kingan SB, et al. De novo assembly of haplotype-resolved genomes with trio binning. Nat Biotechnol. 2018. https://doi.org/10.1038/nbt.4277.

3. Miga KH, Koren S, Rhie A, Vollger MR, Gershman A, Bzikadze A, et al. Telomere-to-telomere assembly of a complete human X chromosome. bioRxiv. 2019. doi:https://doi.org/10.1101/735928.

4. Rice ES, Koren S, Rhie A, Heaton MP, Kalbfleisch TS, Hardy T, et al. Continuous chromosome-scale haplotypes assembled from a single interspecies F1 hybrid of yak and cattle. Gigascience. 2020;9:1-9.

5. Ballouz S, Dobin A, Gillis JA. Is it time to change the reference genome? Genome Biol. 2019;20:159. https://doi.org/10. 1186/s13059-019-1774-4.

6. FAO. The second report on the state of the world's animal genetic resources for food and agriculcure. Rome: Food and Agriculture Organization (FAO); 2015.

7. Daetwyler HD, Capitan A, Pausch H, Stothard P, Van Binsbergen R, Brøndum RF, et al. Whole-genome sequencing of 234 bulls facilitates mapping of monogenic and complex traits in cattle. Nat Genet. 2014;46:858-65. https://doi.org/10.1038/ng.3034.

8. Hayes BJ, Daetwyler HD. 1000 Bull Genomes Project to map simple and complex genetic traits in cattle: applications and outcomes. Annu Rev Anim Biosci. 2019;7:annurev-animal-020518-115024. doi:https://doi.org/10.1146/annurevanimal-020518-115024.

9. Charlier C, Li W, Harland C, Littlejohn M, Coppieters W, Creagh F, et al. NGS-based reverse genetic screen for common embryonic lethal mutations compromising fertility in livestock. Genome Res. 2016;26:1333-41.

10. Bovine Genome Sequencing and Analysis Consortium. The genome sequence of taurine cattle: a window to ruminant biology and evolution. Science (80- ). 2009;324:522-8. https://doi.org/10.1126/science.1169588.

11. Worley K, Gibbs R. Sequencing the bovine genome. Oxford: Wiley-Blackwell; 2012.

12. Crysnanto $D$, Wurmser $C$, Pausch $H$. Accurate sequence variant genotyping in cattle using variation-aware genome graphs. Genet Sel Evol. 2019;51:21. https://doi.org/10.1186/s12711-019-0462-x. 
13. Jansen $S$, Aigner $B$, Pausch $H$, Wysocki M, Eck $S$, Benet-Pagès $A$, et al. Assessment of the genomic variation in a cattle population by re-sequencing of key animals at low to medium coverage. BMC Genomics. 2013;14:446. https://doi.org/ 10.1186/1471-2164-14-446.

14. Kim J, Hanotte O, Mwai OA, Dessie T, Salim B, Diallo B, et al. The genome landscape of indigenous African cattle. Genome Biol. 2017;18:1-14.

15. Koufariotis L, Hayes BJ, Kelly M, Burns BM, Lyons R, Stothard P, et al. Sequencing the mosaic genome of Brahman cattle identifies historic and recent introgression including polled. Sci Rep. 2018;8:17761. https://doi.org/10.1038/s41598-018-35698-5.

16. Van De Geijn B, Mcvicker G, Gilad Y, Pritchard JK. WASP: allele-specific software for robust molecular quantitative trait locus discovery. Nat Methods. 2015;12:1061-3.

17. Paten B, Novak AM, Eizenga JM, Garrison E. Genome graphs and the evolution of genome inference. Genome Res. 2017; 27:665-76. https://doi.org/10.1101/gr.214155.116.

18. Pritt J, Chen N-C, Langmead B. FORGe: prioritizing variants for graph genomes. Genome Biol. 2018;19:220. https://doi. org/10.1186/s13059-018-1595-x.

19. Günther T, Nettelblad C. The presence and impact of reference bias on population genomic studies of prehistoric human populations. PLoS Genet. 2019;15:e1008302.

20. Salavati M, Bush SJ, Palma-Vera S, McCulloch MEB, Hume DA, Clark EL. Elimination of reference mapping bias reveals robust immune related allele-specific expression in crossbred sheep. Front Genet. 2019;10:1-16.

21. Degner JF, Marioni JC, Pai AA, Pickrell JK, Nkadori E, Gilad Y, et al. Effect of read-mapping biases on detecting allele-specific expression from RNA-sequencing data. Bioinformatics. 2009;25:3207-12. https:/doi.org/10.1093/bioinformatics/btp579.

22. Garrison E, Sirén J, Novak AM, Hickey G, Eizenga JM, Dawson ET, et al. Variation graph toolkit improves read mapping by representing genetic variation in the reference. Nat Biotechnol. 2018;36:875-81. https://doi.org/10.1038/nbt.4227.

23. Groza C, Kwan T, Soranzo N, Pastinen T, Bourque G. Personalized and graph genomes reveal missing signal in epigenomic data. Genome Biol. 2020;21:1-22.

24. Sirén J, Garrison E, Novak AM, Paten B, Durbin R. Haplotype-aware graph indexes. Bioinformatics. 2019;36:1-8.

25. Hickey G, Heller D, Monlong J, Sibbesen JA, Sirén J, Eizenga J, et al. Genotyping structural variants in pangenome graphs using the vg toolkit. Genome Biol. 2020;21:1-17.

26. Bhati M, Kadri NK, Crysnanto D, Pausch H. Assessing genomic diversity and signatures of selection in Original Braunvieh cattle using whole-genome sequencing data. BMC Genomics. 2020;21:1-14.

27. Signer-Hasler H, Burren A, Neuditschko M, Frischknecht M, Garrick D, Stricker C, et al. Population structure and genomic inbreeding in nine Swiss dairy cattle populations. Genet Sel Evol. 2017:49:1-13.

28. Auton A, Abecasis GR, Altshuler DM, Durbin RM, Bentley DR, Chakravarti A, et al. A global reference for human genetic variation. Nature. 2015;526:68-74.

29. Tenesa A, Navarro P, Hayes BJ, Duffy DL, Clarke GM, Goddard ME, et al. Recent human effective population size estimated from linkage disequilibrium. Genome Res. 2007;17:520-6.

30. Pausch H, Aigner B, Emmerling R, Edel C, Götz KU, Fries R. Imputation of high-density genotypes in the Fleckvieh cattle population. Genet Sel Evol. 2013;45:1. https://doi.org/10.1186/1297-9686-45-3.

31. Hagger C. Estimates of genetic diversity in the brown cattle population of Switzerland obtained from pedigree information. J Anim Breed Genet. 2005;122:405-13.

32. Grytten I, Rand KD, Nederbragt AJ, Sandve GK. Assessing graph-based read mappers against a novel baseline approach highlights strengths and weaknesses of the current generation of methods. BMC Genomics. 2020;21. https://doi.org/10.1101/538066.

33. Li H. Minimap2: pairwise alignment for nucleotide sequences. Bioinformatics. 2018;34:3094-100.

34. Li H. Aligning sequence reads, clone sequences and assembly contigs with BWA-MEM. arXiv. 2013. http://arxiv.org/abs/1303.3997.

35. Shukla HG, Bawa PS, Srinivasan S. hg19KIndel: ethnicity normalized human reference genome. BMC Genomics. 2019;20: 459. https://doi.org/10.1186/s12864-019-5854-3.

36. Dewey FE, Chen R, Cordero SP, Ormond KE, Caleshu C, Karczewski KJ, et al. Phased whole-genome genetic risk in a family quartet using a major allele reference sequence. PLoS Genet. 2011;7:e1002280. https://doi.org/10.1371/journal.pgen.1002280.

37. Beyer W, Novak AM, Hickey G, Chan J, Tan V, Paten B, et al. Sequence tube maps: making graph genomes intuitive to commuters. Bioinformatics. 2019. https://doi.org/10.1093/bioinformatics/btz597.

38. Li H, Handsaker B, Wysoker A, Fennell T, Ruan J, Homer N, et al. The sequence alignment/map format and SAMtools. Bioinformatics. 2009;25:2078-9. https://doi.org/10.1093/bioinformatics/btp352.

39. Poplin R, Ruano-Rubio V, Depristo MA, Fennell TJ, Carneiro MO, Van Der Auwera GA, et al. Scaling accurate genetic variant discovery to tens of thousands of samples. bioRxiv. 2017. https://doi.org/10.1101/201178.

40. Eggertsson HP, Jonsson H, Kristmundsdottir S, Hjartarson E, Kehr B, Masson G, et al. Graphtyper enables population-scale genotyping using pangenome graphs. Nat Genet. 2017;49:1654-60. https://doi.org/10.1038/ng.3964.

41. Maciuca S, Elias CDO, McVean G, lqbal Z. A natural encoding of genetic variation in a burrows-wheeler transform to enable mapping and genome inference. In: Lecture Notes in Computer Science (including subseries Lecture Notes in Artificial Intelligence and Lecture Notes in Bioinformatics). Springer Verlag; 2016. p. 222-233.

42. Kim D, Paggi JM, Park C, Bennett C, Salzberg SL. Graph-based genome alignment and genotyping with HISAT2 and HISAT-genotype. Nat Biotechnol. 2019:37:907-15. https://doi.org/10.1038/s41587-019-0201-4.

43. Hall SJG. Effective population sizes in cattle, sheep, horses, pigs and goats estimated from census and herdbook data. Animal. 2016;10:1778-85.

44. Leroy G, Mary-Huard T, Verrier E, Danvy S, Charvolin E, Danchin-Burge C. Methods to estimate effective population size using pedigree data: examples in dog, sheep, cattle and horse. Genet Sel Evol. 2013;45:1-10.

45. Bouwman AC, Daetwyler HD, Chamberlain AJ, Ponce CH, Sargolzaei M, Schenkel FS, et al. Meta-analysis of genomewide association studies for cattle stature identifies common genes that regulate body size in mammals. Nat Genet. 2018;50:362-7. https://doi.org/10.1038/s41588-018-0056-5.

46. Crysnanto D, Pausch H. Data for bovine graphs experiments (Version 1.1) [Data set]. 2020. https://doi.org/10.5281/ zenodo.3759712. Accessed 13 July 2020.

47. Liu R, Low WY, Tearle R, Koren S, Ghurye J, Rhie A, et al. New insights into mammalian sex chromosome structure and evolution using high-quality sequences from bovine $X$ and $Y$ chromosomes. BMC Genomics. 2019;20:1-11. https://doi. org/10.1186/s12864-019-6364-z. 
48. Li H, Wren J. Toward better understanding of artifacts in variant calling from high-coverage samples. Bioinformatics. 2014;30:2843-51. https://doi.org/10.1093/bioinformatics/btu356.

49. Malomane DK, Reimer C, Weigend S, Weigend A, Sharifi AR, Simianer H. Efficiency of different strategies to mitigate ascertainment bias when using SNP panels in diversity studies. BMC Genomics. 2018;19:22. https:/doi.org/10.1186/s12864-017-4416-9.

50. Li H, Bloom JM, Farjoun Y, Fleharty M, Gauthier L, Neale B, et al. A synthetic-diploid benchmark for accurate variantcalling evaluation. Nat Methods. 2018;15:595-7. https://doi.org/10.1038/s41592-018-0054-7.

51. Zook JM, McDaniel J, Olson ND, Wagner J, Parikh H, Heaton H, et al. An open resource for accurately benchmarking small variant and reference calls. Nat Biotechnol. 2019:1. doi:https://doi.org/10.1038/s41587-019-0074-6.

52. Rozowsky J, Abyzov A, Wang J, Alves P, Raha D, Harmanci A, et al. AlleleSeq: analysis of allele-specific expression and binding in a network framework. Mol Syst Biol. 2011;7:1-15. https://doi.org/10.1038/msb.2011.54.

53. Sherman RM, Forman J, Antonescu V, Puiu D, Daya M, Rafaels N, et al. Assembly of a pan-genome from deep sequencing of 910 humans of African descent. Nat Genet. 2019;51:30-5. https://doi.org/10.1038/s41588-018-0273-y.

54. Hehir-Kwa JY, Marschall T, Kloosterman WP, Francioli LC, Baaijens JA, Dijkstra LJ, et al. A high-quality human reference panel reveals the complexity and distribution of genomic structural variants. Nat Commun. 2016;7:1-10.

55. Holden LA, Arumilli M, Hytönen MK, Hundi S, Salojärvi J, Brown KH, et al. Assembly and analysis of unmapped genome sequence reads reveal novel sequence and variation in dogs. Sci Rep. 2018;8:1-11.

56. Li R, Li Y, Zheng H, Luo R, Zhu H, Li Q, et al. Building the sequence map of the human pan-genome. Nat Biotechnol. 2010;28:57-62.

57. Rosen BD, Bickhart DM, Schnabel RD, Koren S, Elsik CG, Tseng E, et al. De novo assembly of the cattle reference genome with single-molecule sequencing. Gigascience. 2020;9:1-9.

58. Chaisson MJP, Sanders AD, Zhao X, Malhotra A, Porubsky D, Rausch T, et al. Multi-platform discovery of haplotype-resolved structural variation in human genomes. Nat Commun. 2019;10:1784. https://doi.org/10.1038/s41467-018-08148-z.

59. Alkan C, Coe BP, Eichler EE. Genome structural variation discovery and genotyping. Nat Rev Genet. 2011;12:363-76

60. Chen S, Krusche P, Dolzhenko E, Sherman RM, Petrovski R, Schlesinger F, et al. Paragraph: a graph-based structural variant genotyper for short-read sequence data. Genome Biol. 2019;20.

61. Rakocevic G, Semenyuk V, Lee WP, Spencer J, Browning J, Johnson IJ, et al. Fast and accurate genomic analyses using genome graphs. Nat Genet. 2019;51:354-62. https://doi.org/10.1038/s41588-018-0316-4.

62. Eggertsson HP, Kristmundsdottir S, Beyter D, Jonsson H, Skuladottir A, Hardarson MT, et al. GraphTyper2 enables population-scale genotyping of structural variation using pangenome graphs. Nat Commun. 2019;10:5402. https://doi. org/10.1038/s41467-019-13341-9.

63. Duan Z, Qiao Y, Lu J, Lu H, Zhang W, Yan F, et al. HUPAN: a pan-genome analysis pipeline for human genomes. Genome Biol. 2019;20. https://doi.org/10.1186/s13059-019-1751-y.

64. Beyter D, Ingimundardottir H, Eggertsson HP, Bjornsson E, Kristmundsdottir S, Mehringer S, et al. Long read sequencing of 1,817 Icelanders provides insight into the role of structural variants in human disease. bioRxiv. 2019.

65. Li H, Feng X, Chu C. The design and construction of reference pangenome graphs. Arxiv. 2020. http://arxiv.org/abs/2 003.06079. Accessed 13 July 2020.

66. Baes CF, Dolezal MA, Koltes JE, Bapst B, Fritz-Waters E, Jansen S, et al. Evaluation of variant identification methods for whole genome sequencing data in dairy cattle. BMC Genomics. 2014;15:948. https://doi.org/10.1186/1471-2164-15-948

67. Hofstetter S, Seefried F, Häfliger IM, Jagannathan V, Leeb T, Drögemüller C. A non-coding regulatory variant in the 5'region of the MITF gene is associated with white-spotted coat in Brown Swiss cattle. Anim Genet. 2019;50:27-32.

68. Tarasov A, Vilella AJ, Cuppen E, Nijman IJ, Prins P. Sambamba: fast processing of NGS alignment formats. Bioinformatics. 2015;31:2032-4. https://doi.org/10.1093/bioinformatics/btv098.

69. Browning BL, Browning SR. Genotype imputation with millions of reference samples. Am J Hum Genet. 2016;98:116-26. https://doi.org/10.1016/.j.jhg.2015.11.020.

70. Danecek P, Auton A, Abecasis G, Albers CA, Banks E, DePristo MA, et al. The variant call format and VCFtools. Bioinformatics. 2011;27:2156-8. https://doi.org/10.1093/bioinformatics/btr330.

71. Rausch T, Zichner T, Schlattl A, Stütz AM, Benes V, Korbel JO. DELLY: structural variant discovery by integrated pairedend and split-read analysis. Bioinformatics. 2012;28:333-9.

72. Chang CC, Chow CC, Tellier LCAM, Vattikuti S, Purcell SM, Lee JJ. Second-generation PLINK: Rising to the challenge of larger and richer datasets. Gigascience. 2015;4:1-16.

73. Browning BL, Zhou Y, Browning SR. A one-penny imputed genome from next-generation reference panels. Am J Hum Genet. 2018;103:338-48. https://doi.org/10.1016/j.ajhg.2018.07.015.

74. Haeussler M, Zweig AS, Tyner C, Speir ML, Rosenbloom KR, Raney BJ, et al. The UCSC Genome Browser database: 2019 update. Nucleic Acids Res. 2019;47:D853-8.

75. Yates AD, Achuthan P, Akanni W, Allen J, Allen J, Alvarez-Jarreta J, et al. Ensembl 2020. Nucleic Acids Res. 2020:48:682-8.

76. Smit A, Hubley R, Green P. RepeatMasker Open-4.0. http://www.repeatmasker.org. Accessed 8 Apr 2020.

77. Chen S, Zhou Y, Chen Y, Gu J. Fastp: an ultra-fast all-in-one FASTQ preprocessor. Bioinformatics. 2018;34:1884-90. https:// doi.org/10.1093/bioinformatics/bty 560 .

78. Novak AM, Hickey G, Garrison E, Blum S, Connelly A, Dilthey A, et al. Genome Graphs. bioRxiv 2017. doi:https://doi.org/ $10.1101 / 101378$

79. Linderman MD, Brandt T, Edelmann L, Jabado O, Kasai Y, Kornreich R, et al. Analytical validation of whole exome and whole genome sequencing for clinical applications. BMC Med Genet. 2014;7:20. https://doi.org/10.1186/1755-8794-7-20.

80. Köster J, Rahmann S. Snakemake--a scalable bioinformatics workflow engine. Bioinformatics. 2012;28:2520-2. https://doi. org/10.1093/bioinformatics/bts480.

81. Crysnanto D, Pausch H. Scripts for bovine graphs experiments (Version 1.1). 2020. https://doi.org/10.5281/zenodo. 3763286. Accessed 13 July 2020.

\section{Publisher's Note}

Springer Nature remains neutral with regard to jurisdictional claims in published maps and institutional affiliations. 